\title{
Diagnostic capabilities of spectropolarimetric observations for understanding solar phenomena
}

\section{Zeeman-sensitive photospheric lines}

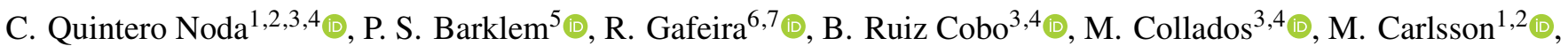 \\ V. Martínez Pillet ${ }^{8}$, D. Orozco Suárez ${ }^{6}$, H. Uitenbroek ${ }^{8}$, and Y. Katsukawa ${ }^{9}{ }^{\circ}$
}

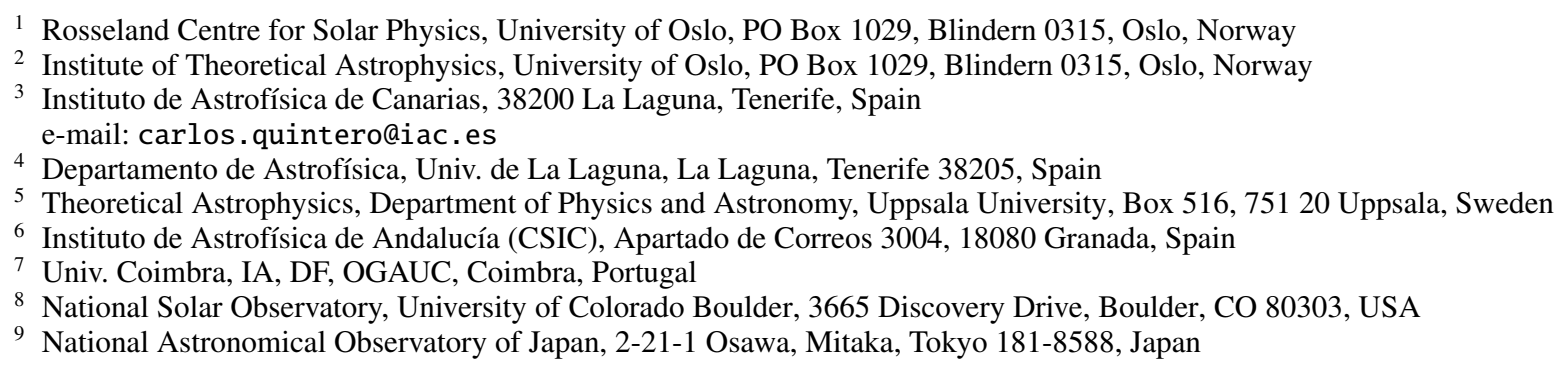

Received 14 February 2020 / Accepted 18 May 2021

\begin{abstract}
Future ground-based telescopes will expand our capabilities for simultaneous multi-line polarimetric observations in a wide range of wavelengths, from the near-ultraviolet to the near-infrared. This creates a strong demand to compare candidate spectral lines to establish a guideline of the lines that are most appropriate for each observation target. We focused in this first work on Zeemansensitive photospheric lines in the visible and infrared. We first examined their polarisation signals and response functions using a 1D semi-empirical atmosphere. Then we studied the spatial distribution of the line core intensity and linear and circular polarisation signals using a realistic 3D numerical simulation. We ran inversions of synthetic profiles, and we compared the heights at which we obtain a high correlation between the input and the inferred atmosphere. We also used this opportunity to revisit the atomic information we have on these lines and computed the broadening cross-sections due to collisions with neutral hydrogen atoms for all the studied spectral lines. The results reveal that four spectral lines stand out from the rest for quiet-Sun and network conditions: Fe I 5250.2, 6302,8468 , and $15648 \AA$. The first three form higher in the atmosphere, and the last line is mainly sensitive to the atmospheric parameters at the bottom of the photosphere. However, as they reach different heights, we strongly recommend using at least one of the first three candidates together with the Fe I $15648 \AA$ line to optimise our capabilities for inferring the thermal and magnetic properties of the lower atmosphere.
\end{abstract}

Key words. Sun: magnetic fields - techniques: polarimetric - atomic data - Sun: photosphere - radiative transfer

\section{Introduction}

The decade 2020 represents an excellent opportunity for performing spectropolarimetric observations at high spatial resolution. Ground-based telescopes such as the Daniel K. Inouye Solar Telescope (DKIST, Keil et al. 2011) and the European Solar Telescope (EST, Collados et al. 2013) will mean a significant leap in the diameters of the primary mirror and consequently, in spatial resolution for work at the diffraction limit. The third flight of the SUNRISE balloon-borne telescope (Solanki et al. 2010; Barthol et al. 2011) is scheduled for 2022, and although it has a lower spatial resolution, it will work at almost atmospheric-free conditions and observe the Sun continuously for several days. In all these missions, polarimetric measurements are the primary target. Most importantly, simultaneous observations of multiple spectral lines will be the baseline of most observing configurations.

A reference compendium of spectral lines optimised for the observing targets of interest is needed. Supporting studies are required that compare these lines at identical conditions and for various physical scenarios. We have recurred to these studies in the past. For example, Norton et al. (2006) provide information based on which we can decide which spectral lines are to be included in the Helioseismic and Magnetic Imager (Schou et al. 2012) on board of the Solar Dynamics Observatory (Pesnell et al. 2012). A series of publications started with Leenaarts et al. (2013) to support the Interface Region Imaging Spectrograph (De Pontieu et al. 2014) mission, and Quintero Noda et al. (2016, 2017a,b, 2018) to select spectral lines of the SUNRISE Chromospheric Infrared spectroPolarimeter (Katsukawa et al. 2020) and the upgraded version of the Imaging Magnetograph eXperiment (Martínez Pillet et al. 2011) instrument.

The aim of this work and following papers is to do something similar, but with a much broader scope, focusing on DKIST and EST, as we continue to support SUNRISE. The main difference is that this research project needs to cover most of the spectral range from the near-UV to the near-IR, that is, $3800-15000 \AA$, which implies a vast number of possible lines that we cannot study in detail. We therefore opt to examine candidate lines that 
are included in the documentation of these future telescopes. Most of the transitions are well known and have been analysed in the past. Still, one of the critical targets of these telescopes is to observe the most capable lines together, increasing the height resolution of the observations. However, even if we restrict ourselves to the best candidate lines, many spectral lines with different formation heights and capabilities remain from which the atmospheric information may be inferred. We therefore narrow down the target of this work even more to spectral lines that are highly magnetically sensitive and form in the photosphere. After this, we will continue with publications that compare chromospheric lines at identical conditions and non-magnetic spectral lines. Our aims are mainly twofold. On the one hand, we wish to provide a reference of which spectral regions should be observed in an optimum way for the teams that are responsible for the instrument development. On the other hand, we wish to generate a compendium of spectral lines that will help observers of these future missions to tailor their observing proposals based on their science targets.

\section{Method}

\subsection{Synthesis of the Stokes profiles}

We make use of the code called Stokes inversion based on response functions (SIR; Ruiz Cobo \& del Toro Iniesta 1992) to synthese the full Stokes vector. The code assumes local thermodynamic equilibrium (LTE).

We used two types of atmospheric models, 1D semiempirical atmospheres, and 3D realistic numerical simulations. In the first case, we employed the Harvard-Smithsonian Reference Atmosphere (HSRA, Gingerich et al. 1971) to compute the dependence of the polarisation signals on the field strength and the response functions (RF; e.g., Landi Degl'Innocenti \& Landi Degl'Innocenti 1977) to perturbations of the atmospheric parameters. In the second case, we used the snapshot 385 of the enhanced network simulation described in Carlsson et al. (2016) and developed with the Bifrost code (Gudiksen et al. 2011). The snapshot covers a surface of $24 \times 24 \mathrm{Mm}^{2}$ with a pixel size of $48 \mathrm{~km}$, while the vertical domain extends from 2.4 Mm below to $14.4 \mathrm{Mm}$ above the average optical depth unity at $\lambda=5000 \AA$. Although the simulated scenario is simpler than recent numerical simulations such as were presented in Hansteen et al. (2017, 2019), and Cheung et al. (2019), it contains a configuration with strong network patches and quiet areas (see Fig. 1). These structures allow ascertaining the capabilities of the spectral lines for quiet-Sun spectropolarimetry. Moreover, the simulation is publicly available ${ }^{1}$, so that our work can be extended with additional lines that can be compared with our results.

For both studies we assumed disk centre observations, that is, $\mu=1$, where $\mu=\cos (\theta)$ and $\theta$ is the heliocentric angle. We used the abundance values of the different atomic species given in Asplund et al. (2009). We defined a spectral sampling of $10 \mathrm{~mA}$. We did not degrade the Stokes profiles with any macroturbulence correction or by adding the effect of noise or spatial resolution. Finally, we did not include any microturbulence enhancement for the HSRA atmosphere and the enhanced network simulation. Previous studies (Leenaarts et al. 2009) mentioned the lack of small-scale velocities in the Bifrost simulation that should be accounted for because otherwise, the polarisation signals would be artificially enhanced (see de la Cruz Rodríguez et al. 2012). See also the detailed discussion presented in Carlin

\footnotetext{
1 http://sdc.uio.no/search/simulations
}

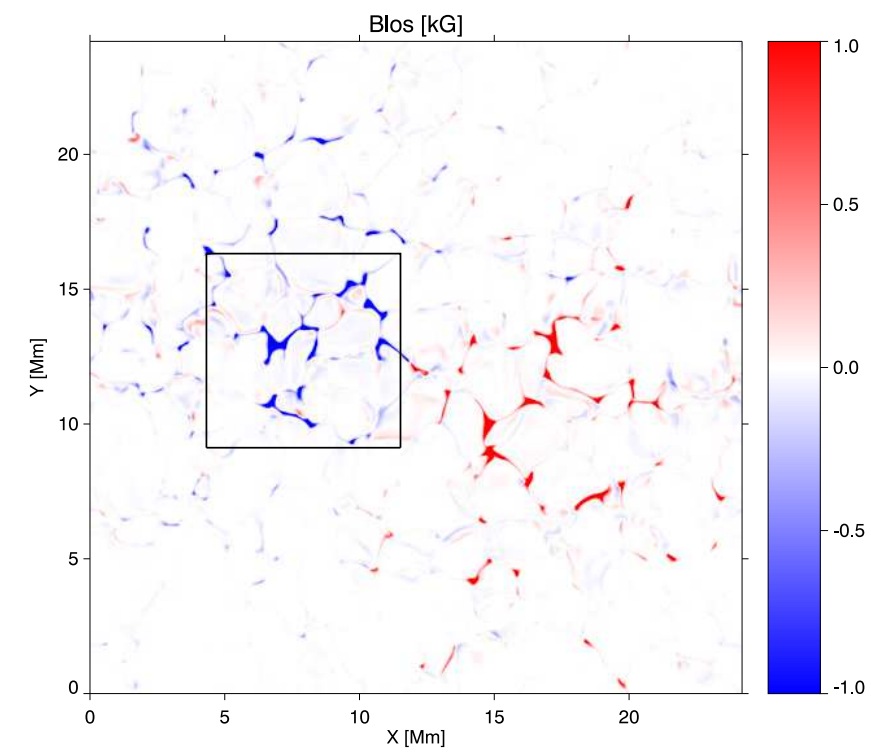

Fig. 1. Longitudinal field strength at the $5000 \AA$ continuum optical depth $\log \tau=0$ in the snapshot 385 from the BIFROST enhanced network simulation.

\& Bianda (2017) for a more in-depth analysis of the impact of a microturbulence enhancement on the polarisation signals for this simulation. However, we are more interested here in the relative differences between the spectral lines of interest than in comparing with actual observations.

\subsection{Spectral lines}

We performed a thorough selection of spectral lines based on different sources, mainly the EST, DKIST, and SUNRISE science requirement documents, and Table 1 in Smitha \& Solanki (2017). We tried to cover as many candidate lines for photospheric Zeeman spectropolarimetry as possible, but we may have missed some of them. We are therefore open to suggestions for future publications.

Table 1 contains the spectroscopic information of the spectral lines in which we are interested. We extracted this information from the NIST database (Kramida et al. 2018) as the primary source. However, there are cases where the $\log g f$ is not specified, therefore we used the R. Kurucz (Kurucz \& Bell 1995) database instead (see asterisks). We followed the same steps as Quintero Noda et al. (2018) to compute the sensitivity indices to linear and circular polarisation as explained in the monograph of Landi Degl'Innocenti \& Landolfi (2004). The advantage of using these effective values is that we also considered the spectral line wavelength and line core intensity. Therefore they provide a more accurate estimation of the sensitivity of the spectral lines to the Zeeman effect (see e.g., the study presented in Martínez González et al. 2008). Quintero Noda et al. (2018) described how each of the necessary elements was computed to obtain the first-order $\bar{g}$ and second-order $\bar{G}$ Landé factors, as well as the sensitivity indices $s_{Q}$ and $s_{V}$. We therefore refer to this work for more information and to Landi Degl'Innocenti \& Landolfi (2004) for a complete description. The solar atlas used for the computation was observed by Delbouille et al. (1973) and provided by the BASS2000 solar archive ${ }^{2}$. The continuum was estimated locally (in wavelength), and the intensities were 
C. Quintero Noda et al.: Diagnostic capabilities of spectropolarimetric observations for understanding solar phenomena. I.

Table 1. Polarisation sensitivity indices $s_{Q}$ and $s_{V}$ (Landi Degl'Innocenti \& Landolfi 2004) for a selection of spectral lines.

\begin{tabular}{|c|c|c|c|c|c|c|c|c|c|c|c|c|c|c|}
\hline Atom & $\lambda[\AA]$ & $\log (g f)$ & $L_{1}$ & $L_{2}$ & $g_{1}$ & $g_{2}$ & $\bar{g}$ & $s$ & $d$ & $\bar{G}$ & $I\left(\lambda_{0}\right)[\mathrm{AU}]$ & $d_{\mathrm{c}}$ & $s_{Q}$ & $s_{V}$ \\
\hline $\mathrm{Fe} I$ & 5247.0501 & -4.946 & ${ }^{5} \mathrm{D}_{2}$ & ${ }^{7} \mathrm{D}_{3}^{\mathrm{o}}$ & 1.50 & 1.75 & 2.00 & 18.00 & -6.00 & 3.98 & 2808 & 0.719 & 1.509 & 3.152 \\
\hline $\mathrm{Fe} I$ & 5250.2086 & -4.938 & ${ }^{5} \mathrm{D}_{0}$ & ${ }^{7} \mathrm{D}_{1}^{\mathrm{o}}$ & 0.00 & 3.00 & 3.00 & 2.00 & -2.00 & 9.00 & 2841 & 0.715 & 2.252 & 7.094 \\
\hline $\mathrm{Fe} \mathrm{I}$ & 5250.6456 & -2.181 & ${ }^{5} \mathrm{P}_{2}$ & ${ }^{5} \mathrm{P}_{3}^{\mathrm{o}}$ & 1.83 & 1.67 & 1.50 & 18.00 & -6.00 & 2.24 & 2028 & 0.796 & 1.254 & 1.967 \\
\hline $\mathrm{Fe} I$ & 6173.3352 & -2.880 & ${ }^{5} \mathrm{P}_{1}$ & ${ }^{5} \mathrm{D}_{0}^{\mathrm{o}}$ & 2.50 & 0.00 & 2.50 & 2.00 & 2.00 & 6.25 & 3738 & 0.626 & 1.933 & 5.966 \\
\hline $\mathrm{Fe} \mathrm{I}$ & 6301.5008 & -0.718 & ${ }^{5} \mathrm{P}_{2}^{\mathrm{o}}$ & ${ }^{5} \mathrm{D}_{2}$ & 1.83 & 1.50 & 1.67 & 12.00 & 0.00 & 2.52 & 2780 & 0.722 & 1.520 & 2.890 \\
\hline $\mathrm{Fe} I$ & 6302.4932 & $-1.131 *$ & ${ }^{5} \mathrm{P}_{1}^{\mathrm{o}}$ & ${ }^{5} \mathrm{D}_{0}$ & 2.50 & 0.00 & 2.50 & 2.00 & 2.00 & 6.25 & 3443 & 0.656 & 2.066 & 6.511 \\
\hline Ni I & 6767.7700 & -2.170 & ${ }^{1} \mathrm{~S}_{0}$ & ${ }^{3} \mathrm{P}_{1}^{\mathrm{o}}$ & 0.00 & 1.50 & 1.50 & 2.00 & -2.00 & 2.25 & 3630 & 0.637 & 1.293 & 2.626 \\
\hline $\mathrm{Fe} I$ & 6820.3715 & -1.290 & ${ }^{5} \mathrm{P}_{1}^{\mathrm{o}}$ & ${ }^{5} \mathrm{P}_{2}$ & 2.50 & 1.83 & 1.50 & 8.00 & -4.00 & 2.18 & 6453 & 0.355 & 0.726 & 1.439 \\
\hline $\mathrm{Fe} I$ & 6842.6854 & -1.290 & ${ }^{5} \mathrm{P}_{1}^{\mathrm{o}}$ & ${ }^{5} \mathrm{P}_{1}$ & 2.50 & 2.50 & 2.50 & 4.00 & 0.00 & 6.25 & 6589 & 0.341 & 1.167 & 3.992 \\
\hline $\mathrm{Fe} I$ & 8468.4069 & -2.072 & ${ }^{5} \mathrm{P}_{1}$ & ${ }^{5} \mathrm{P}_{1}^{\mathrm{o}}$ & 2.50 & 2.50 & 2.50 & 4.00 & 0.00 & 6.25 & 3643 & 0.636 & 2.691 & 11.40 \\
\hline $\mathrm{Fe} \mathrm{I}$ & 8514.0716 & -2.229 & ${ }^{5} \mathrm{P}_{2}$ & ${ }^{5} \mathrm{P}_{2}^{\mathrm{o}}$ & 1.83 & 1.83 & 1.83 & 12.00 & 0.00 & 3.36 & 3813 & 0.619 & 1.928 & 6.027 \\
\hline $\mathrm{Fe} I$ & 10783.051 & $-2.571^{*}$ & ${ }^{3} \mathrm{P}_{0}$ & ${ }^{3} \mathrm{P}_{1}^{\mathrm{o}}$ & 0.00 & 1.50 & 1.50 & 2.00 & -2.00 & 2.25 & 6877 & 0.312 & 1.010 & 3.268 \\
\hline Si I & 10786.856 & -0.365 & ${ }^{3} \mathrm{P}_{1}^{\mathrm{o}}$ & ${ }^{3} \mathrm{P}_{0}$ & 1.50 & 0.00 & 1.50 & 2.00 & 2.00 & 2.25 & 3906 & 0.609 & 1.972 & 6.382 \\
\hline $\mathrm{Fe} \mathrm{I}$ & 15534.246 & $-0.585^{*}$ & ${ }^{5} \mathrm{D}_{1}$ & ${ }^{5} \mathrm{P}_{2}^{\mathrm{o}}$ & 1.50 & 1.83 & 2.00 & 8.00 & -4.00 & 3.98 & 6606 & 0.339 & 2.109 & 13.04 \\
\hline $\mathrm{Fe} \mathrm{I}$ & 15542.083 & $-0.457 *$ & ${ }^{5} \mathrm{D}_{1}$ & ${ }^{5} \mathrm{D}_{0}^{\mathrm{o}}$ & 1.50 & 0.00 & 1.50 & 2.00 & 2.00 & 2.25 & 7157 & 0.284 & 1.326 & 6.181 \\
\hline $\mathrm{Fe} \mathrm{I}$ & 15648.514 & $-0.714^{*}$ & ${ }^{7} \mathrm{D}_{1}$ & ${ }^{7} \mathrm{D}_{1}^{\mathrm{o}}$ & 3.00 & 3.00 & 3.00 & 4.00 & 0.00 & 9.00 & 7003 & 0.300 & 2.814 & 26.42 \\
\hline
\end{tabular}

Notes. From left to right, we show the atomic species, the line core wavelength in air, $\log (g f)$, lower and upper level spectroscopic notation, the Landé factor of the lower and upper level, the effective first-order Landé factor, $s$ and $d$ (see Quintero Noda et al. 2018), the effective second-order Landé factor, the line core intensity (extracted from the solar atlas of Delbouille et al. 1973), the normalised line depth $d_{\mathrm{c}}$, and the circular and linear polarisation sensitivity index. We define $\lambda_{\text {ref }}=5000 \AA$, and the continuum corresponds to $I_{\mathrm{c}}=10000$ (in arbitrary units, AU) for all the lines.

normalized so that the continuum was $100 \%$ (value 10000 arbitrary units, AU). In our case, $I_{\mathrm{c}}=10000 \mathrm{AU}$ for all the spectral lines.

We summarise some context information and publications that have used these spectral lines to explain why we considered them here. We only describe here the lines in detail later below, while the so-called auxiliary lines are included in Table 1 for completeness and are justified in the next section.

We start with the Fe I $5250.2 \AA$ line, which has been widely used for polarimetric observations since the early 1970s, for instance, by Stenflo (1973), because it has an effective Landé factor of 3 and also strongly depends on the temperature. Modern instrumentation such as the Imaging Magnetograph eXperiment (Martínez Pillet et al. 2011) on board the SUNRISE balloon-borne telescope (Solanki et al. 2010; Barthol et al. 2011) also observes it to further improve our knowledge of the magnetic field in the lower atmosphere (see e.g., the recent review of Bellot Rubio \& Orozco Suárez 2019). The next photospheric candidate is the Fe I $6173 \AA$ spectral line, which has become one of the best transitions for space magnetographs because it has a clean continuum and no blends (Norton et al. 2006). It is observed routinely by the Helioseismic and Magnetic Imager (Schou et al. 2012) on board the Solar Dynamics Observatory (Pesnell et al. 2012) and will also be observed by the Polarimetric and Helioseismic Imager (Solanki et al. 2020) on board the Solar Orbiter mission (Müller \& Marsden 2013). The following spectral line is the Fe I $6302.5 \AA$, which was extensively recorded by the Advanced Stokes Polarimeter (Elmore et al. 1992) in the 1990s and was later the main spectral line for analysing the solar photosphere at high spatial resolution from space through the observations made by the Spectropolarimeter (Lites et al. 2013) of the Solar optical telescope (Tsuneta et al. 2008) on board the Hinode spacecraft
(Kosugi et al. 2007). The next transition we studied is the Ni I $6768 \AA$ line, one of the most famous spectral lines for helioseismology and the analysis of large-scale photospheric magnetism. It was routinely observed by the Michelson Doppler Imager (Scherrer et al. 1995) on board the SoHo mission (Domingo et al. 1995), and it is recorded daily by the Global Oscillation Network Group project (Harvey et al. 1996) to study the solar internal structure through helioseismology. The Fe I $6842 \AA$ spectral line has a high sensitivity to the magnetic field with $\bar{g}=2.5$, and it also forms in the low photosphere (e.g., Smitha \& Solanki 2017). The next transition is the Fe I $8468 \AA$, which is located relatively close to the Ca II 8498 and $8542 \AA$ chromospheric lines. Hence, it is part of the critical spectral lines of the SUNRISE Chromospheric Infrared spectro-Polarimeter (Katsukawa et al. 2020) that will simultaneously observe the $770 \mathrm{~nm}$ region (Quintero Noda et al. 2017b) and the $850 \mathrm{~nm}$ window described in Quintero Noda et al. (2017a). The line is strong, and has an effective Landé factor of 2.5 as well. The Fe I $10783 \AA$ line has a lower magnetic field sensitivity, $\bar{g}=1.5$, but is a target line for future missions because it is well suited for sunspot observations as it does not show indications of blends in the umbra (Balthasar \& Gömöry 2008). The next transition on the list, that is, Fe I $15534 \AA$, with $\bar{g}=2.0$, is a good candidate for line-ratio techniques (Smitha \& Solanki 2017) and can be observed at present, for instance, with the GRIS spectrograph (Collados et al. 2012) installed on the Gregor telescope (Schmidt et al. 2012). Finally, GRIS as well as its predecessor (the Tenerife Infrared Polarimeter, Collados et al. 2007) has routinely observed the Fe I $15648 \AA$ line, the last selected transition for this work. This line is one of the few candidates that has a Lande factor of 3.0, and it is one of the lines of excellence for quiet-Sun observations (see e.g., Bellot Rubio \& Orozco Suárez 2019, for more information). 
Table 2. Atomic information used to compute the collisional broadening parameters.

\begin{tabular}{|c|c|c|c|c|c|c|c|c|c|c|c|c|c|}
\hline Atom & $\lambda[\AA]$ & Lower configuration and term & Upper configuration and term & $E_{\text {lower }}\left[\mathrm{cm}^{-1}\right]$ & $E_{\text {lower }}^{\text {limit }}\left[\mathrm{cm}^{-1}\right]$ & $E_{\text {upper }}\left[\mathrm{cm}^{-1}\right]$ & $E_{\text {upper }}^{\text {limit }}\left[\mathrm{cm}^{-1}\right]$ & $l_{\text {low }}$ & $l_{\text {upp }}$ & $J_{\text {low }}$ & $J_{\text {upp }}$ & $\sigma[\mathrm{AU}]$ & $\alpha$ \\
\hline $\mathrm{Fe} I$ & 5247.0501 & $3 d^{6} 4 s^{2} a{ }^{5} D$ & $3 d^{6}\left({ }^{5} D\right) 4 s 4 p\left({ }^{3} P^{o}\right) z^{7} D^{o}$ & 704.007 & 63737.704 & 19757.032 & 63737.704 & 0 & 1 & 2 & 3 & 205 & 0.255 \\
\hline $\mathrm{Fe} I$ & 5250.2086 & $3 d^{6} 4 s^{2} a{ }^{5} D$ & $3 \mathrm{~d}^{6}\left({ }^{5} \mathrm{D}\right) 4 \mathrm{~s} 4 \mathrm{p}\left({ }^{3} \mathrm{P}^{\mathrm{o}}\right) \mathrm{z}^{7} \mathrm{D}^{\mathrm{o}}$ & 978.074 & 63737.704 & 20019.635 & 63737.704 & 0 & 1 & 0 & 1 & 206 & 0.254 \\
\hline $\mathrm{Fe} I$ & 5250.6456 & $3 d^{7}\left({ }^{4} P\right) 4 s$ a ${ }^{5} P$ & $3 \mathrm{~d}^{6}\left({ }^{5} \mathrm{D}\right) 4 \mathrm{~s} 4 \mathrm{p}\left({ }^{1} \mathrm{P}^{\mathrm{o}}\right)$ y ${ }^{5} \mathrm{P}^{\mathrm{o}}$ & 17726.988 & 76933.840 & 36766.966 & 63578.161 & 0 & 1 & 2 & 3 & 343 & 0.266 \\
\hline $\mathrm{Fe} I$ & 6173.3352 & $3 \mathrm{~d}^{7}\left({ }^{4} \mathrm{P}\right) 4 \mathrm{~s}$ a ${ }^{5} \mathrm{P}$ & $3 d^{7}\left({ }^{4} F\right) 4 p$ y ${ }^{5} D^{0}$ & 17927.382 & 76933.840 & 34121.603 & 65738.010 & 0 & 1 & 1 & 0 & 279 & 0.245 \\
\hline $\mathrm{Fe} I$ & 6301.5008 & $3 \mathrm{~d}^{6}\left({ }^{5} \mathrm{D}\right) 4 \mathrm{~s} 4 \mathrm{p}\left({ }^{3} \mathrm{P}^{\mathrm{o}}\right) \mathrm{z}^{5} \mathrm{P}^{\mathrm{o}}$ & $3 d^{6}\left({ }^{5} D\right) 4 s\left({ }^{6} D\right) 5 s e^{5} D$ & 29469.024 & 63737.704 & 45333.875 & 63737.704 & 1 & 0 & 2 & 2 & 834 & 0.242 \\
\hline $\mathrm{Fe} I$ & 6302.4932 & $3 d^{6}\left({ }^{5} D\right) 4 s 4 p\left({ }^{3} P^{o}\right) z^{5} P^{o}$ & $3 d^{6}\left({ }^{5} D\right) 4 s\left({ }^{6} D\right) 5 s e^{5} D$ & 29732.736 & 63737.704 & 45595.086 & 63737.704 & 1 & 0 & 1 & 0 & 850 & 0.239 \\
\hline $\mathrm{Ni} I$ & 6767.7700 & $3 d^{10}{ }^{1} S$ & $3 d^{9}\left({ }^{2} D\right) 4 p^{3} P^{o}$ & 14728.840 & 61619.77 & 29500.674 & 61619.77 & 2 & 1 & 0 & 1 & 301 & 0.241 \\
\hline $\mathrm{Fe} I$ & 6820.3715 & $3 \mathrm{~d}^{6}\left({ }^{5} \mathrm{D}\right) 4 \mathrm{~s} 4 \mathrm{p}\left({ }^{1} \mathrm{P}^{\mathrm{o}}\right)$ y ${ }^{5} \mathrm{P}^{\mathrm{o}}$ & $3 d^{6}\left({ }^{5} D\right) 4 s\left({ }^{6} D\right) 4 d e^{5} P$ & 37409.555 & 63737.704 & 52067.469 & 63737.704 & 1 & 2 & 1 & 2 & 897 & 0.282 \\
\hline $\mathrm{Fe} I$ & 6842.6854 & $3 \mathrm{~d}^{6}\left({ }^{5} \mathrm{D}\right) 4 \mathrm{~s} 4 \mathrm{p}\left({ }^{1} \mathrm{P}^{\mathrm{o}}\right)$ y ${ }^{5} \mathrm{P}^{\mathrm{o}}$ & $3 d^{6}\left({ }^{5} D\right) 4 s\left({ }^{6} D\right) 4 d e^{5} P$ & 37409.555 & 63737.704 & 52019.669 & 63737.704 & 1 & 2 & 1 & 1 & 891 & 0.281 \\
\hline $\mathrm{Fe} I$ & 8468.4069 & $3 \mathrm{~d}^{7}\left({ }^{4} \mathrm{P}\right) 4 \mathrm{~s}$ a ${ }^{5} \mathrm{P}$ & $3 \mathrm{~d}^{6}\left({ }^{5} \mathrm{D}\right) 4 \mathrm{~s} 4 \mathrm{p}\left({ }^{3} \mathrm{P}^{\mathrm{o}}\right) \mathrm{z}^{5} \mathrm{P}^{\mathrm{o}}$ & 17927.382 & 76933.840 & 29732.736 & 63737.704 & 0 & 1 & 1 & 1 & 259 & 0.245 \\
\hline Ti I & 8468.4700 & $3 \mathrm{~d}^{3}\left({ }^{2} \mathrm{G}\right) 4 \mathrm{~s} \mathrm{a}^{3} \mathrm{G}$ & $3 \mathrm{~d}^{2}\left({ }^{1} \mathrm{D}\right) 4 \mathrm{~s} 4 \mathrm{p}\left({ }^{3} \mathrm{P}^{\mathrm{o}}\right) \mathrm{x}^{3} \mathrm{~F}^{\mathrm{o}}$ & 15220.393 & 63912.060 & 27025.659 & 63578.161 & 0 & 1 & 5 & 4 & 268 & 0.252 \\
\hline $\mathrm{FeI}$ & 8514.0716 & $3 \mathrm{~d}^{7}\left({ }^{4} \mathrm{P}\right) 4 \mathrm{~s}$ a ${ }^{5} \mathrm{P}$ & $3 \mathrm{~d}^{6}\left({ }^{5} \mathrm{D}\right) 4 \mathrm{~s} 4 \mathrm{p}\left({ }^{3} \mathrm{P}^{\mathrm{o}}\right) \mathrm{z}^{5} \mathrm{P}^{\mathrm{o}}$ & 17726.988 & 76933.840 & 29469.024 & 63737.704 & 0 & 1 & 2 & 2 & 257 & 0.246 \\
\hline $\mathrm{Fe} I$ & 10783.051 & $3 \mathrm{~d}^{7}\left({ }^{2} \mathrm{P}\right) 4 \mathrm{~s} \mathrm{c}^{3} \mathrm{P}$ & $3 \mathrm{~d}^{6}\left({ }^{5} \mathrm{D}\right) 4 \mathrm{~s} 4 \mathrm{p}\left({ }^{3} \mathrm{P}^{\mathrm{o}}\right) \mathrm{z}^{3} \mathrm{P}^{\mathrm{o}}$ & 25091.599 & 81857.423 & 34362.873 & 63737.704 & 0 & 1 & 0 & 1 & 306 & 0.245 \\
\hline Si I & 10786.856 & $3 s^{2} 3 p 4 p^{3} D 2$ & $3 \mathrm{~s}^{2} 3 \mathrm{p} 4 \mathrm{~d}^{3} \mathrm{~F}^{\mathrm{o}}$ & 48102.323 & 66035.000 & 57372.297 & 66035.000 & 1 & 2 & 2 & 2 & 1341 & 0.302 \\
\hline $\mathrm{Fe} I$ & 15534.246 & $3 d^{6}\left({ }^{5} D\right) 4 s\left({ }^{6} D\right) 5 s e^{5} D$ & $3 \mathrm{~d}^{6}\left({ }^{5} \mathrm{D}\right) 4 \mathrm{~s}\left({ }^{6} \mathrm{D}\right) 5 \mathrm{p} \mathrm{u}^{5} \mathrm{P}^{\mathrm{o}}$ & 45509.152 & 63737.704 & 51944.784 & 63737.704 & 0 & 1 & 1 & 2 & 1180 & 0.237 \\
\hline $\mathrm{Fe} I$ & 15542.083 & $3 \mathrm{~d}^{6}\left({ }^{5} \mathrm{D}\right) 4 \mathrm{~s}\left({ }^{6} \mathrm{D}\right) 5 \mathrm{~s}$ e ${ }^{5} \mathrm{D}$ & $3 \mathrm{~d}^{6}\left({ }^{5} \mathrm{D}\right) 4 \mathrm{~s}\left({ }^{6} \mathrm{D}\right) 5 \mathrm{p} \mathrm{t}{ }^{5} \mathrm{D}^{0}$ & 45509.152 & 63737.704 & 51941.540 & 63737.704 & 0 & 1 & 1 & 0 & 1179 & 0.237 \\
\hline $\mathrm{Fe} I$ & 15648.514 & $3 d^{6}\left({ }^{5} D\right) 4 s\left({ }^{6} D\right) 5 s e^{7} D$ & $3 \mathrm{~d}^{6}\left({ }^{5} \mathrm{D}\right) 4 \mathrm{~s}\left({ }^{6} \mathrm{D}\right) 5 \mathrm{p} \mathrm{n}^{7} \mathrm{D}^{\mathrm{o}}$ & 43763.980 & 63737.704 & 50152.619 & 63737.704 & 0 & 1 & 1 & 1 & 974 & 0.229 \\
\hline $\mathrm{Fe} I$ & 15652.873 & $3 d^{6}\left({ }^{5} D\right) 4 s\left({ }^{6} D\right) 4 d f^{7} D$ & $3 d^{6} 4 s\left({ }^{6} D_{9 / 2}\right) 4 f\left({ }^{2}[7 / 2]^{\circ}\right)$ & 50377.908 & 63737.704 & 56764.767 & 63737.704 & 2 & 3 & 5 & 4 & 1428 & 0.330 \\
\hline
\end{tabular}

Notes. From left to right, we list the atom species, transition wavelength, the dominant configuration and term designation for the lower and upper levels, the energy of the lower level, the series limit energy for that level, the energy of the upper level, the series limit energy for that level, the orbital angular momentum quantum numbers of the optical electron in the lower and upper levels, the total angular momentum quantum numbers of the lower and upper levels, and finally the calculated line broadening cross-section $\sigma$ due to hydrogen atom impact at a collision velocity of $10^{4} \mathrm{~m} \mathrm{~s}^{-1}$ and corresponding velocity dependence parameter $\alpha$. The units for the wavelength are $\AA$, the energy is listed in $\mathrm{cm}^{-1}$, and atomic units for the cross-section $\sigma$, respectively. The velocity parameter $\alpha$ is dimensionless.

\subsection{Auxiliary spectral lines}

A few of the spectral lines included in Table 1 are not strictly the best candidates for Zeeman polarimetry. Their effective Landé factor is not as high as that of the other lines, for example, or they do not present particular properties in specific magnetic configurations. However, they are similar to those that are very sensitive to the magnetic field and were used in the past to estimate, for instance, the field strength of quiet-Sun observations (Stenflo 1973). Stenflo (1973) computed the ratio of the Fe I 5247 and $5250.2 \AA$ lines because the atomic levels that produced their transitions are identical, except for the total angular momentum values. This technique allowed them to infer the field strength from the Stokes $V$ amplitude ratio because the difference between the two lines should be the Lande factor. It was therefore customary to observe them together, and we expect that some observers will do the same with future facilities. We therefore also included the Fe I 8468 and $8515 \AA$ as they represent a similar case. Furthermore, the $8515 \AA$ line lies between the two Ca II 8498 and $8542 \AA$ lines and would represent an excellent photospheric complement to these two chromospheric lines. We also added the two Fe I 10783 and Si I $10786 \AA$ lines as they are close enough to be observed together and can complement each other. Finally, we list in Table 2 additional lines that fall close to the target spectral lines of this work and are usually observed together, for instance, Fe I 5250.6, 6301.5, or $15652 \AA$.

\subsection{Fe। $8468.4069 \AA$ and Til $8468.4700 \AA$}

In previous works, we presented the Fe I $8468.4069 \AA$ as one of the most capable photospheric spectral lines (e.g., Quintero Noda et al. 2017a). It should therefore be included in this study as well. However, when we presented the preliminary results of this work at the National Solar Observatory, we received some helpful feedback from Alexandra Tritschler. She mentioned that there is a TiI transition close to the iron line at $8468.4700 \AA$ that might modify its profile shape, mainly for cool atmospheres where titanium lines are usually enhanced (see e.g., the review of Solanki 2003). We therefore decided to quickly compare the results obtained by synthesising only the iron transition or both spectral lines in this publication. The results are plotted in Fig. 2, where we used the HSRA atmosphere adding a constant magnetic field (top) and the cool umbra model presented in Collados et al. (1994) (bottom). When the Ti I line is included, the Stokes profiles in the HSRA atmosphere are slightly modified. The perturbation is mainly in the red wing of the iron line as the titanium transition falls at $63.1 \mathrm{~m} \AA$ from its line core. In the case of the cool model, the differences are more significant, and the perturbation appears to be closer to the centre of the iron line. From now on, we therefore need to include the titanium line when synthesising or inverting the Fe I $8468.4069 \AA$ transition (as we do in this publication).

\subsection{Collisions with neutral hydrogen}

The broadening of the spectral lines by collisions with neutral hydrogen atoms is computed using the Anstee, Barklem and O'Mara (ABO) theory (e.g., Anstee \& O'Mara 1995; Barklem \& O'Mara 1997), specifically the abo-cross calculator code (Barklem et al. 2015), which interpolates in pre-computed tables of line broadening parameters. A description of how to use the code can be found in Barklem et al. (1998). For clarity, we describe two examples below, one that is straightforward, and another that involves a two-electron excitation. We show in Table 2 the data we used for the transitions of interest and the resulting calculated values of the line broadening parameters, the cross-section $\sigma$ at a collision velocity of $10^{4} \mathrm{~m} \mathrm{~s}^{-1}$, and the velocity parameter $\alpha$. See Barklem et al. (1998) for example for an explanation of these parameters and the formula for calculating the line widths. We include the target lines of this work and the additional transitions that fall close to the spectral lines of interest.

We start with the straightforward example of the Fe I $5250.2 \AA$ transition. We extracted the basic information from the NIST database, including the energy of each level $\left(E_{\text {lower }}\right.$ and $\left.E_{\text {upper }}\right)$ and the orbital angular momentum of the optical electron 
C. Quintero Noda et al.: Diagnostic capabilities of spectropolarimetric observations for understanding solar phenomena. I.
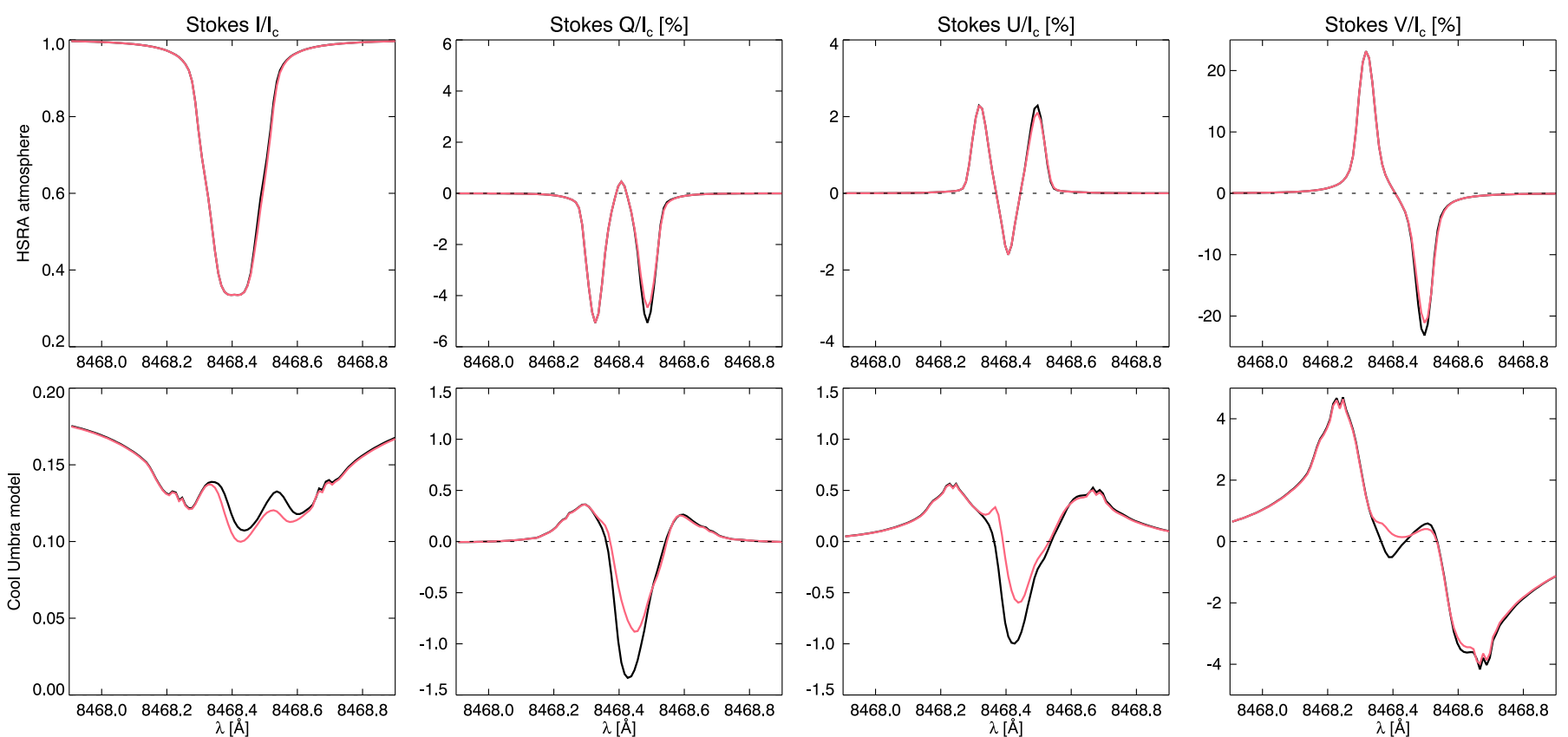

Fig. 2. Comparison of the synthesis of the Fe I $8468.4069 \AA$ Alone (black) and the synthesis of this line blended with Ti I $8468.4700 \AA$ (red). Upper row: results using the HSRA semi-empirical atmosphere with a magnetic field of $500 \mathrm{G}$, and 45 and $70^{\circ}$ of magnetic inclination and azimuth, respectively. Lower row: synthetic profiles from the cool-umbra model atmosphere.

$(l)$. The total angular momentum $(J)$ was not used in the calculation, but was extracted for identification purposes. The remaining input parameters were the series limits for the configurations of each level $E_{\text {limit }}$, which defines the binding energy of the optical electron (see Barklem et al. 1998, for a detailed description and examples). For the two levels involved in this transition, the parent configuration is $3 \mathrm{~d}^{6} 4 \mathrm{~s}$, corresponding to the ground configuration and level of Fe II. Thus, $E_{\text {limit }}$ is simply the ionisation energy of Fe I, that is, $63737.704 \mathrm{~cm}^{-1}$. In the case of the Fe I $5250.6 \AA$ transition, we have the same situation for the upper level: After removing the optical electron, the parent configuration is again $3 \mathrm{~d}^{6} 4 \mathrm{~s}$, and so the series limit energy is also $E_{\text {limit }}=$ $63737.704 \mathrm{~cm}^{-1}$. However, in the lower level of the transition, the parent $3 d^{7}{ }^{4} \mathrm{P}$ corresponds to an excited level of Fe II; that is, at the Fe I level, two electrons are excited, the optical electron and a core electron. The binding energy of the optical electron must account for this excited core. Thus the series limit energy should be the sum of the ionisation energy for Fe I and the energy of this level of Fe II (relative to the ground state of Fe II) as this is the level remaining if the optical electron is removed, thus defining the binding energy. NIST gives a term energy for $3 \mathrm{~d}^{74} \mathrm{P}$ of $13196.137 \mathrm{~cm}^{-1}$, and thus the series energy of the lower level is $E_{\text {limit }}=63737.704+13196.137=76933.840 \mathrm{~cm}^{-1}$. We chose to use the term value because selecting a parent with a specific $J$ or a mixture of $J$ values is not justified by the theory, which ignores spin and angular momentum coupling with the core. Furthermore, this would change the calculation only very little, which is a negligible error relative to the overall accuracy of the theory.

Some of the lines fell just outside the bounds of the tables included in the code; for these transitions, specific calculations were performed. The case of Ni I $6768 \AA$ requires special mention. The lower state has very tightly bound equivalent valence electrons in d orbitals, the wavefunction for which cannot be calculated with the Coulomb wavefunction method employed in the $\mathrm{ABO}$ theory. The broadening of a spectral line is usually dom- inated by the upper level, with some interference effects due to the lower level. To calculate the broadening of this line, including some estimate of the interference due to the lower level, we therefore assumed that the lower level was spherically symmetric (s state), allowing $\mathrm{ABO}$ codes to be employed, and the value we calculated is given in Table 2. Ignoring the lower state completely leads to a change (increase) of about $7 \%$, indicating that the lower level indeed contributes only little to the broadening through interference effects.

As a final note, in the case of the SIR code, the crosssection $\sigma$ needs to be provided in cgs units, that is, $\mathrm{cm}^{2}$. We therefore need to multiply the $\sigma$ values given in the table by $a_{0}^{2}=2.80028 \times 10^{-17} \mathrm{~cm}^{2}$.

\section{Spectral line analysis based on 1D semi-empirical atmospheres}

\subsection{Maximum polarisation signals}

To compute the maximum polarisation signals, we started with the HSRA atmosphere, including a magnetic field constant with height. Then we modified its field strength using $1 \mathrm{G}$ steps from 10 to $1000 \mathrm{G}$. As we planned to estimate the transversal and longitudinal signals separately, we performed the computation with a magnetic field inclination $(\gamma)$ of $90^{\circ}$ first and then with $\gamma=0^{\circ}$. In both cases, the magnetic field azimuth is equal to $70^{\circ}$.

The top panel of Fig. 3 displays the total linear polarisation signals (computed as $L P=\sqrt{Q^{2}+U^{2}}$ ). The Fe I 6842, 10783 , $15534 \AA$, and Ni I $6768 \AA$ lines are the weakest lines. Then, the Fe I 6173, and $6302 \AA$ spectral lines show similar signals that are always stronger than the previous signals. Lastly, another three lines show the strongest signals and correspond to Fe I 5250.2, 8468 , and $15648 \AA$. These results agree with the indices presented in Table 1, indicating that we need to take the Landé factor, the line-to-continuum absorption ratio, and the wavelength 

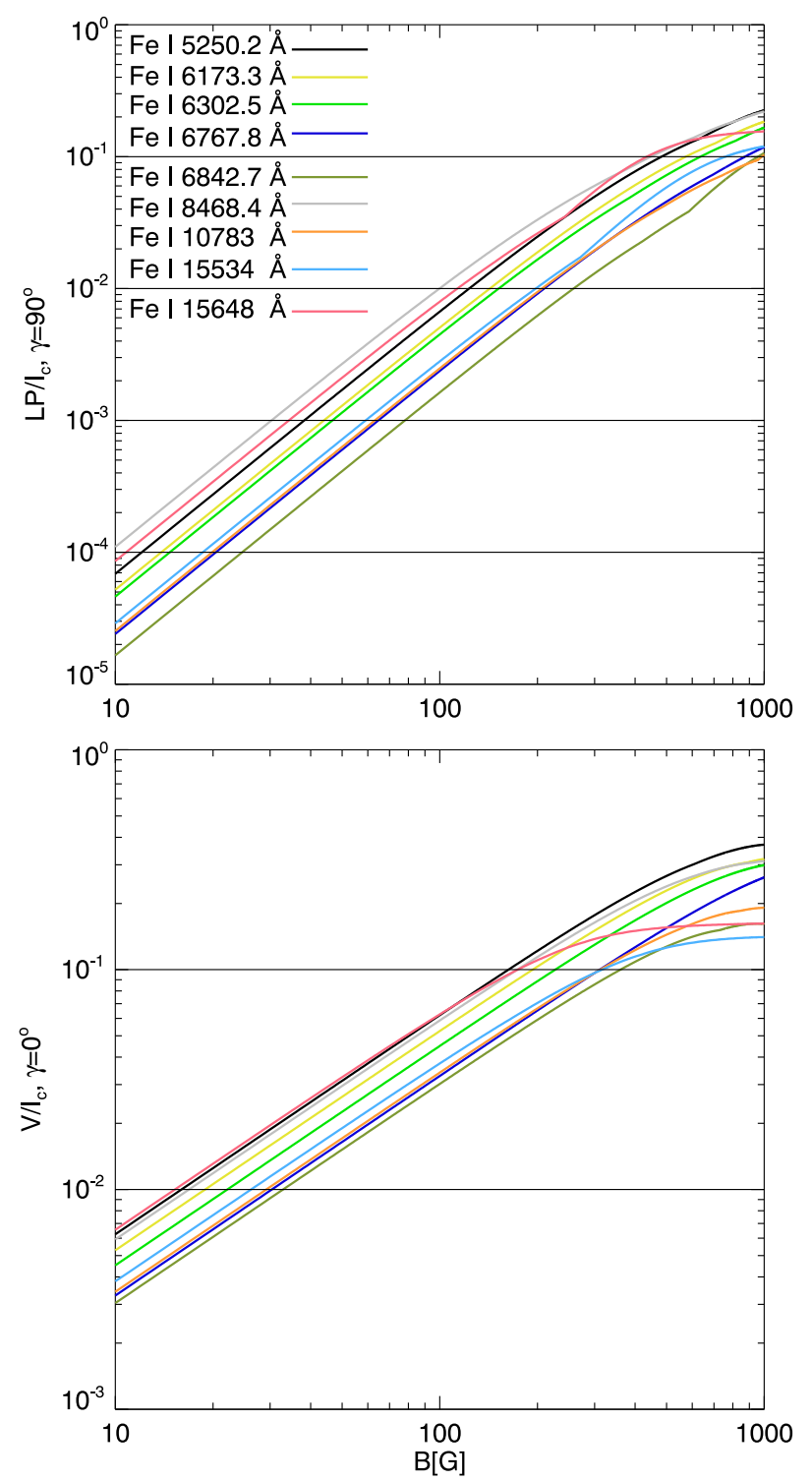

Fig. 3. Maximum linear $\left(L P=\max \left(\sqrt{Q^{2}+U^{2}}\right)\right)$ and circular $(V=$ $\max (|V|))$ polarisation signals for the selected nine most Zeemansensitive photospheric lines (see legend in the top panel). We use the HSRA atmosphere and vary the field strength from 10 to $1000 \mathrm{G}$ (see abscissas). Upper panel: results when the inclination is $90^{\circ}$ (horizontal magnetic field with respect to the solar surface), and lower panel: results for a magnetic field that is parallel to the line of sight, i.e., an inclination of $0^{\circ}$. The azimuth is always equal to $70^{\circ}$.

range into which the transition falls in our selection of spectral lines into consideration (see also the detailed discussion in Cabrera Solana et al. 2005). In the case of the last three lines, we can expect linear polarisation signals around $1 \times 10^{-3}$ of $I_{\mathrm{c}}$ for a reference horizontal magnetic field of $30 \mathrm{G}$.

The circular polarisation signals show a similar behaviour (bottom panel of Fig. 3), although now the Fe I $6173 \AA$ transition is closer to the best three lines mentioned before. The infrared lines (light blue and red) reach the strong-field regime earlier than the others. However, their signals are already stronger than $1 \times 10^{-1}$ of $I_{\mathrm{c}}$, so that this property will not affect their observability, even for noisy observations. Finally, all the studied lines generate circular polarisation signals stronger than $1 \times 10^{-3}$ of $I_{\mathrm{c}}$ for a vertical field of $10 \mathrm{G}$.

\subsection{Response functions to perturbations in the atmospheric parameters}

We computed the response functions (RF, Landi Degl'Innocenti \& Landi Degl'Innocenti 1977) to perturbations in the atmospheric parameters for the nine most sensitive spectral lines studied before. We assumed LTE conditions, so that the SIR code can provide the analytical RFs for any given atmosphere. We used the HSRA model, adding a magnetic field of $500 \mathrm{G}$, and the inclination and azimuth were equal to 45 and $70^{\circ}$, respectively.

To reduce the content of this work, we followed two approaches. We present the $2 \mathrm{D}(\lambda-\log \tau)^{3}$ Stokes $V$ RF to changes in the field strength and a more compact way of comparing the RF plotting its maximum values in a $1 \mathrm{D}$ plot. We show the first case in Fig. 4, where all the spectral lines display an antisymmetric pattern. When the field strength is modified, the amplitude of the Stokes $V$ profile changes equally (in the absence of velocity asymmetries) for the negative and positive lobes. In terms of height, some spectral lines are sensitive to the magnetic field only at lower layers, that is, Fe I 6842, 10 783, 15 534, and $15648 \AA$. The remaining spectral lines reach higher layers up to $\log \tau=-3.5$. Interestingly, the Fe I $15648 \AA$ transition appears to reach deeper layers than the other lines. As a side note, the infrared lines at 1.5 micron show multiple lobes because they already achieved the saturation regime with a field strength of $500 \mathrm{G}$.

It is also useful to study the vertical stratification of the RF at specific wavelengths as was shown, for instance, by Ruiz Cobo \& del Toro Iniesta (1992), Cabrera Solana et al. (2005), and Fossum \& Carlsson (2005). For this reason, we display in Fig. 5 a 1D plot computing the maximum of the RF functions to perturbations in different physical parameters. In this case, we used the four Stokes parameters, so that we can compare the sensitivity of the spectral lines to the three components of the magnetic field vector. For each optical depth, we picked the highest value of the four Stokes parameters at any wavelength of the spectrum.

Starting with the temperature, that is, with the leftmost panel, all the lines at lower layers contribute significantly. The Fe I 5250.2, 6173, 6302, and $8468 \AA$ and Ni I $6768 \AA$ lines extend to upper layers and reach up to $\log \tau=-4$. The LOS velocity RF shows a central peak for all the lines at about $\log \tau=-1.0$, and again the Fe I 5250.2 and $8468 \AA$ lines reach highest in the atmosphere. The Ni I $6768 \AA$ shows a high sensitivity to the LOS velocity as well, comparable to that of the Fe I 5250.2 A transition. A similar behaviour is found for the three components of the magnetic field, although in this case, the Ni transition is less sensitive than the two iron lines mentioned above. In the case of the field strength and azimuth, the dominant RF comes from $\mathrm{Fe}$ I $15648 \AA$ (red), and its peak is located at the bottom of the photosphere. This spectral line also reaches lower in the atmosphere for the three components of the magnetic field.

\section{Spectral line analysis based on 3D realistic simulations}

We highlight in Fig. 1 the selected Field of View (FOV) with solid lines. We chose this region because it contains one of the enhanced network concentrations, but also weakly magnetised areas. In the following, we describe the height stratification of

\footnotetext{
3 We always refer to the continuum optical depth at $5000 \AA$ when using
} $\log \tau$. 
C. Quintero Noda et al.: Diagnostic capabilities of spectropolarimetric observations for understanding solar phenomena. I.
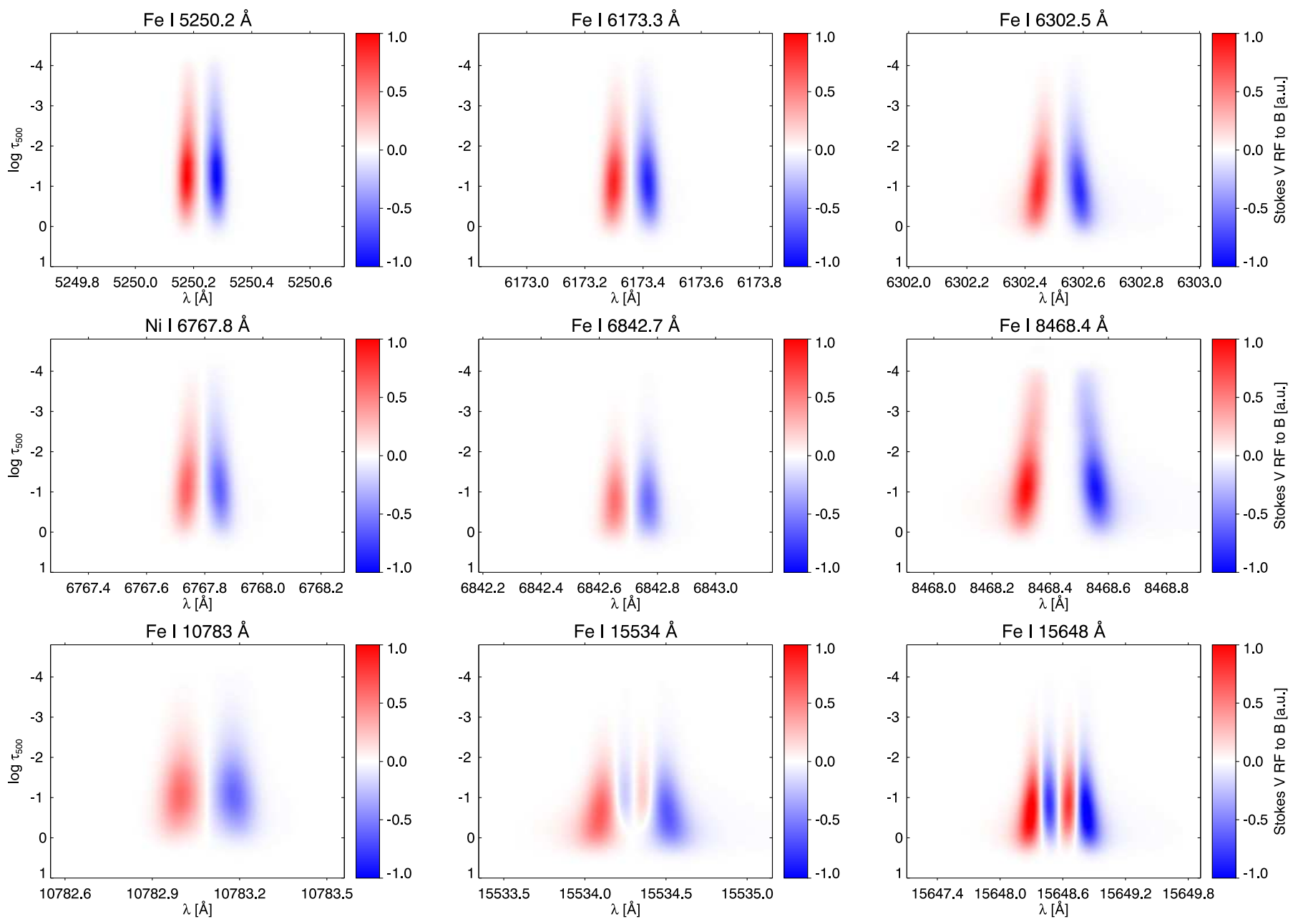

Fig. 4. Two-dimensional plot of the Stokes $V$ RF to changes in the field strength. Each panel displays the results for one of the selected spectral lines. White areas designate regions of no sensitivity to changes in the atmospheric parameters, and red and blue indicate opposite signs of the RF. All panels are normalised to the maximum of the Fe I 5250.2 A Stokes $V$ RF.
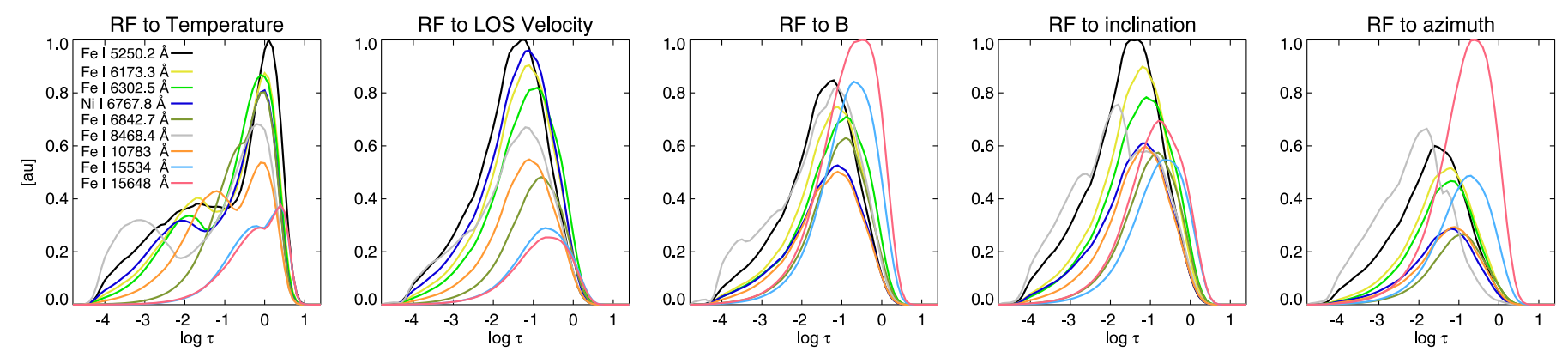

Fig. 5. Maximum RF, from left to right, for the temperature, LOS velocity, and the three components of the magnetic field vector. Each line corresponds to the RF maximum value for the four Stokes parameters and all the computed wavelengths for the selected spectral lines (see colours and the legend in the leftmost panel). RFs are normalised to the maximum value of the nine spectral lines for each atmospheric parameter.

the atmospheric parameters in this region. However, before this, we explain why we transformed the original atmosphere from gas pressure and geometrical height to electronic pressure and optical depth. Ideally, the original parameters should be used because the simulation was run solving the equation of state, and therefore the relation between the atmospheric parameters is consistent for the whole range of heights. However, because we plan to compare the inversion results with the original atmosphere, we preferred to transform everything to optical depth values, which are required as input for SIR, and work from there.

\subsection{Bifrost-enhanced network simulation}

We used snapshot 385, which has been extensively employed in the past for a variety of studies. For example, it was used to characterise spectral lines or estimate the capabilities of the new generation of NLTE radiative transfer codes (among others, Štěpán \& Trujillo Bueno 2016; de la Cruz Rodríguez et al. 2016, 2019; Sukhorukov \& Leenaarts 2017; Bjørgen et al. 2018; Milić \& van Noort 2018; da Silva Santos et al. 2018). We show in Fig. 6 the spatial distribution of the temperature, LOS velocity, 

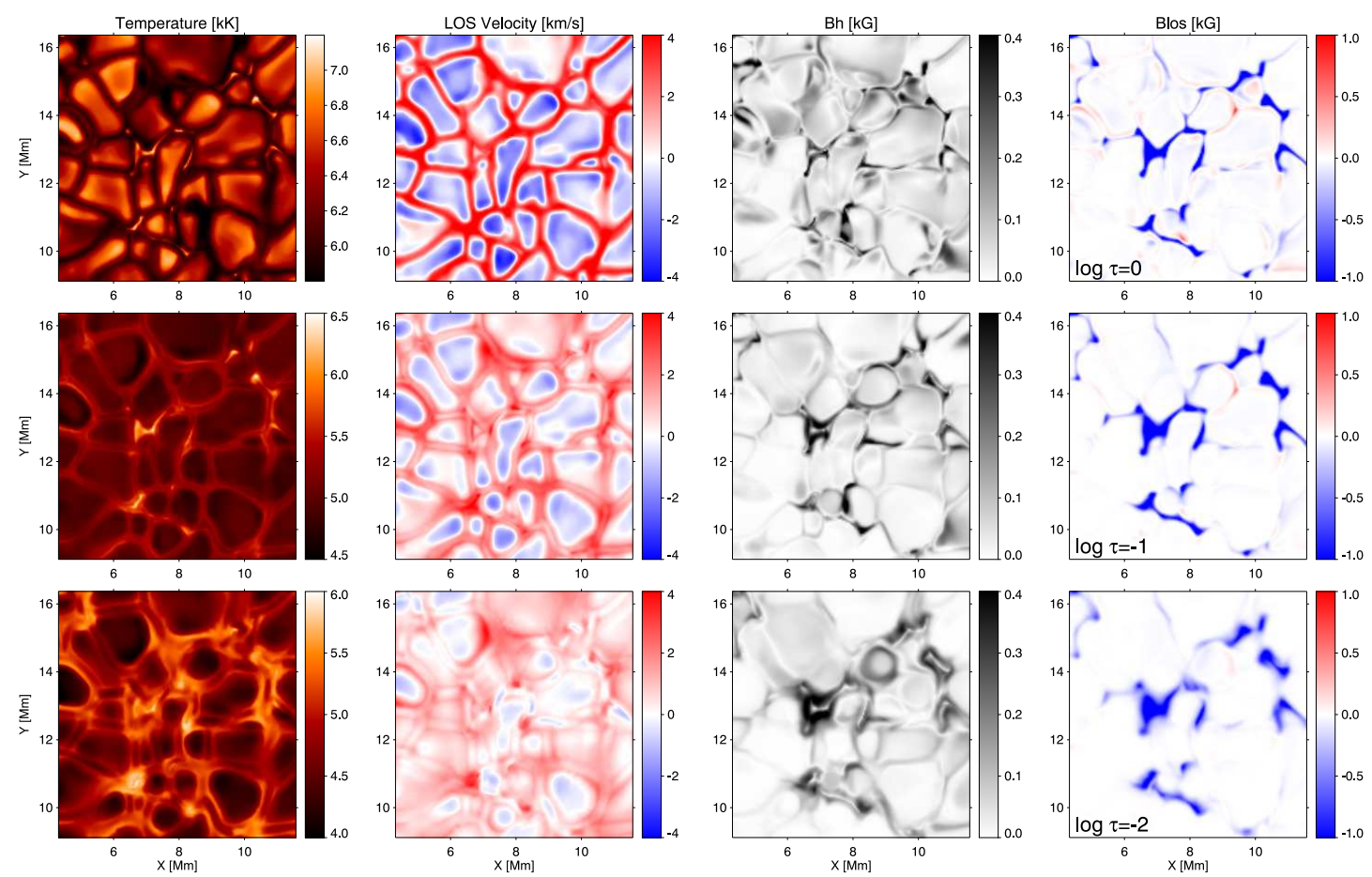

Fig. 6. Snapshot 385 from the BIFROST enhanced network simulation. From left to right: temperature, LOS velocity, and horizontal and longitudinal magnetic field. The rows display different atmospheric layers at $\log \tau=[0,-1,-2]$. The selected FOV corresponds to the region highlighted in Fig. 1.

and the horizontal and longitudinal components of the magnetic field. We chose to display three optical depths (we always refer to the optical depth at $5000 \AA$ ) at $\log \tau=[0,-1,-2]$ because the lines studied in this work are thought to form mostly in between these layers.

Starting with the temperature (leftmost column), the granulation pattern at lower heights with bright intergranular areas coincides with strong magnetic network concentrations. The upper layers display the reverse granulation pattern, with intergranules showing higher temperatures than granules, while the magnetic bright points are still hotter than their surroundings. The spatial distribution of the LOS velocities also shows the granulation pattern at lower heights, and then velocities that fade away at upper layers. In the case of the horizontal component of the magnetic field, we have narrow concentrations of more than $500 \mathrm{G}$ that expand with height. These concentrations are in general located in the surroundings of the enhanced network patches. The longitudinal component is dominated by a single magnetic polarity that occupies broader areas at higher atmospheric layers. The longitudinal component can reach up to $2000 \mathrm{G}$ in the low photosphere, although these values only correspond to the enhanced network areas. The rest of the FOV is characterised by weaker magnetic fields of less than $50 \mathrm{G}$.

\subsection{Spatial distribution of signals}

We show in Fig. 7 the line core intensity of the nine selected spectral lines synthesised with SIR with the configuration explained in Sect. 2. Some lines trace the temperature distribution at lower heights, partially resembling the granulation, such as Fe I 6842, 15 534, and $15648 \AA$. For all of them, we also see the bright features corresponding to the network patches. Then, the Fe I 5250, 6173, 6302, 8468, and $10783 \AA$ and Ni I $6767.8 \AA$ lines trace the temperature at higher photospheric layers, close to the pattern found at $\log \tau=-2$. The visible lines are very similar between them, while Fe I $10783 \AA$ shows more defined structures that would indicate a lower height of formation. The infrared Fe I $8468 \AA$ shows the opposite behaviour, with a more diffused pattern that could be associated with a higher formation height, similar to what we found for the RF analysis.

We present in Fig. 8 the spatial distribution of maximum linear polarisation signals. We saturated the colour scale to $4 \%$ of the local continuum intensity, that is, 0.04 of $I_{\mathrm{c}}$. Weaker lines such as Fe I 6842, 10783 , and $15534 \AA$ and Ni I $6767.8 \AA$ do not reach the saturation value for most of the selected field of view, indicating that they are less suitable for observations with low magnetic activity. On the other hand, Fe I 5250, 6173, 6302,8468 , and $15648 \AA$ reach the bar scale saturation in various areas, for instance at the edges of the network patches. Of the five lines, Fe I 5250 and $8468 \AA$ are similar in terms of spatial distribution and the amplitude of the signals, with the latter showing the highest amplitude. They also display linear polarisation signals inside the large granule at $[9,14] \mathrm{Mm}$. These signals correspond to the horizontal magnetic field at higher layers, see $\log \tau=-2$ in Fig. 6. This explains why they are not present in the Fe I $15648 \AA$ line. However, the infrared line shows stronger polarisation signals in different locations such as $[8,11]$ or $[5.3,15.7] \mathrm{Mm}$.

The results for the maximum circular polarisation signals are shown in Fig. 9. In this case, we saturated the colour bar to $20 \%$ of the local continuum intensity, that is, 0.20 of $I_{\mathrm{c}}$. The Fe I 6842, 10783 , and $15534 \AA$ transitions are again weaker than the others, and they never reach the colour saturation. However, in this case, the Fe I $15648 \AA$ line also shows a similar spatial distribution because the infrared transition reaches the polarisation saturation regime (see also Fig. 3) earlier than the lines in the visible. The Stokes profiles are entirely split, that is, the three Zeeman components are resolved, since about $500 \mathrm{G}$. In 

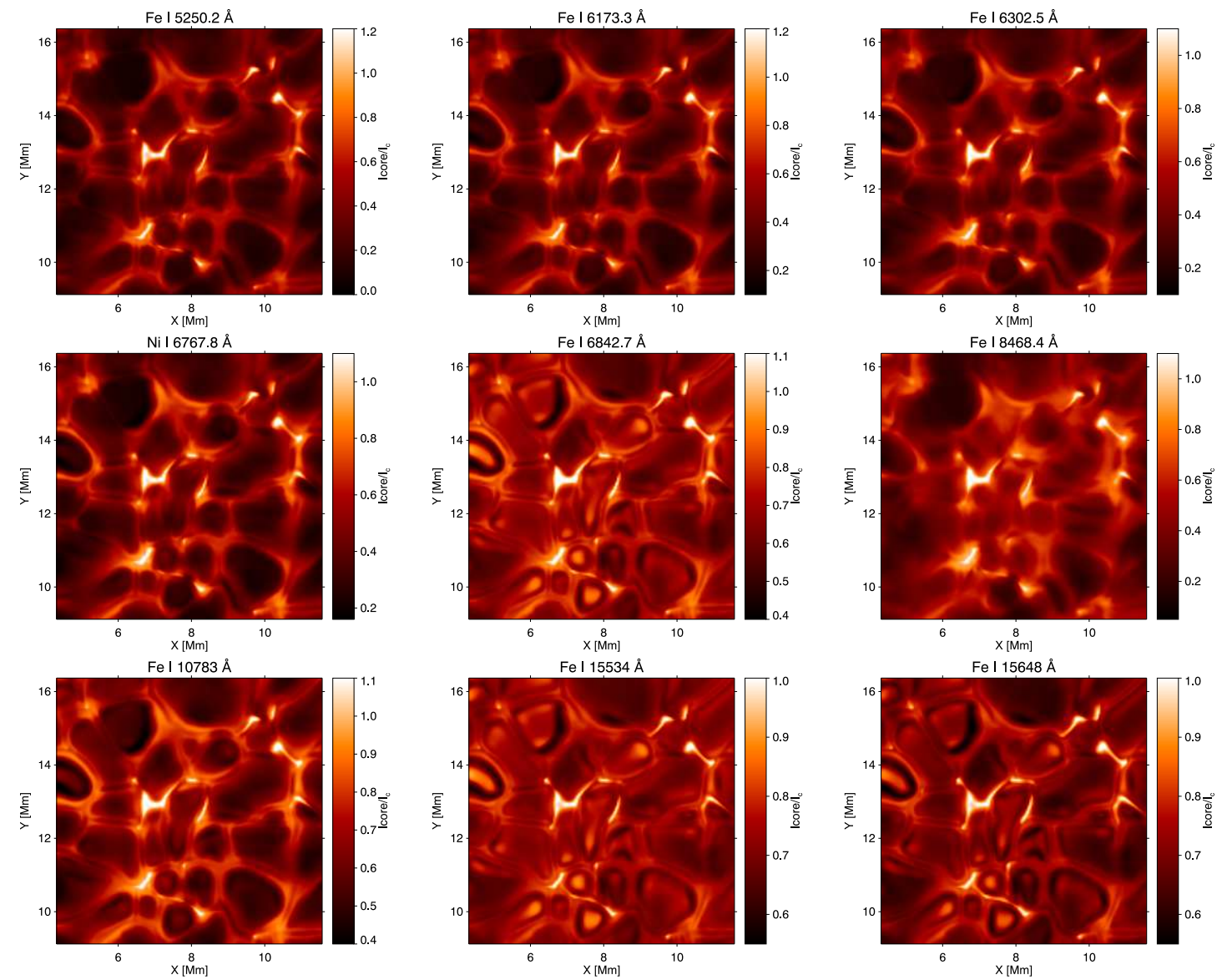

Fig. 7. Spatial distribution of line core intensity signals for the lines of interest. The selected FOV corresponds to the highlighted area in Fig. 1 that is described in Fig. 6.

other words, magnetic fields stronger than the mentioned value will increase the separation of the two $\sigma$ components, while the Stokes $V$ amplitude remains almost constant. This behaviour is partially detected for the visible Fe I $5250 \AA$ line but only in the most active magnetic patch at $[6.3,12.7] \mathrm{Mm}$. For the remaining FOV, the spatial distribution of signals is similar for the Fe I 5250, 6173, 6302, and $8468 \AA$ spectral lines. The latter seems to be sensitive to slightly higher layers, with the polarisation signals occupying more extensive areas. Finally, we also find that the Ni I $6767.8 \AA$ transition shows significant Stokes $V$ signals, comparable to those of Fe I $6302 \AA$, for example, confirming that it is a good candidate for measuring longitudinal fields.

\subsection{Inversions of the Stokes profiles}

We wish to improve our comparison of photospheric lines running inversions of the full Stokes vector. The aim is to estimate how much information we recover when we fit synthetic spectra. We used the profiles from the FOV highlighted in Fig. 1, and we inverted them with the SIR code. We fitted each spectral line separately because we aimed to compare how much information we extracted from each one. We are not interested at this moment in simulating a particular observation, therefore we opted not to include any instrumental degradation or noise.

The inversion was made with SIR using a single magnetic component parametrised with five nodes in temperature, three for the LOS velocity, magnetic field strength, and inclination, and two nodes for the magnetic field azimuth. We did not invert the microturbulence or the macroturbulence, which are both null and equal to the value used for the synthesis. We ran the inversion in parallel using the method described in Sect. 5 of Gafeira et al. (2021), initialising each pixel with three different atmospheres. All of them are modifications of the HSRA model we used in previous sections with different magnetic field configurations. After the three runs, the code automatically picked the solution that produced a better $\chi^{2}$ value.

The inversion process provides the stratification with height of various atmospheric parameters. This information is obtained for each pixel of the FOV highlighted in Fig. 1. This 3D information is inferred for each spectral line of interest, a total of nine transitions. This means that comparing the results would require multiple figures with a format similar to Figs. 7-9. We therefore decided to choose a different approach in this work. We computed the correlation of the original and the inferred atmosphere for a given atmospheric parameter at a given optical depth over the entire FOV $(150 \times 150$ pixels $)$ highlighted in Fig. 1. We computed the correlation for all the optical depths that we have in the inverted atmosphere, obtaining an estimate of how close to the original atmosphere is to the inverted one on average over the complete FOV. Thus, we simplified the visualisation to a single height-dependent vector, that is, the correlation, for each atmospheric parameter and each spectral line.

We present in Fig. 10 the results of the correlation between the atmospheric parameters over the entire FOV for each spectral line using the same colour code as in Fig. 5. Starting with the temperature (leftmost panel), we can detect a high correlation (almost 1 ) in a wide range of heights, $\log \tau=[0,-2.0]$, 

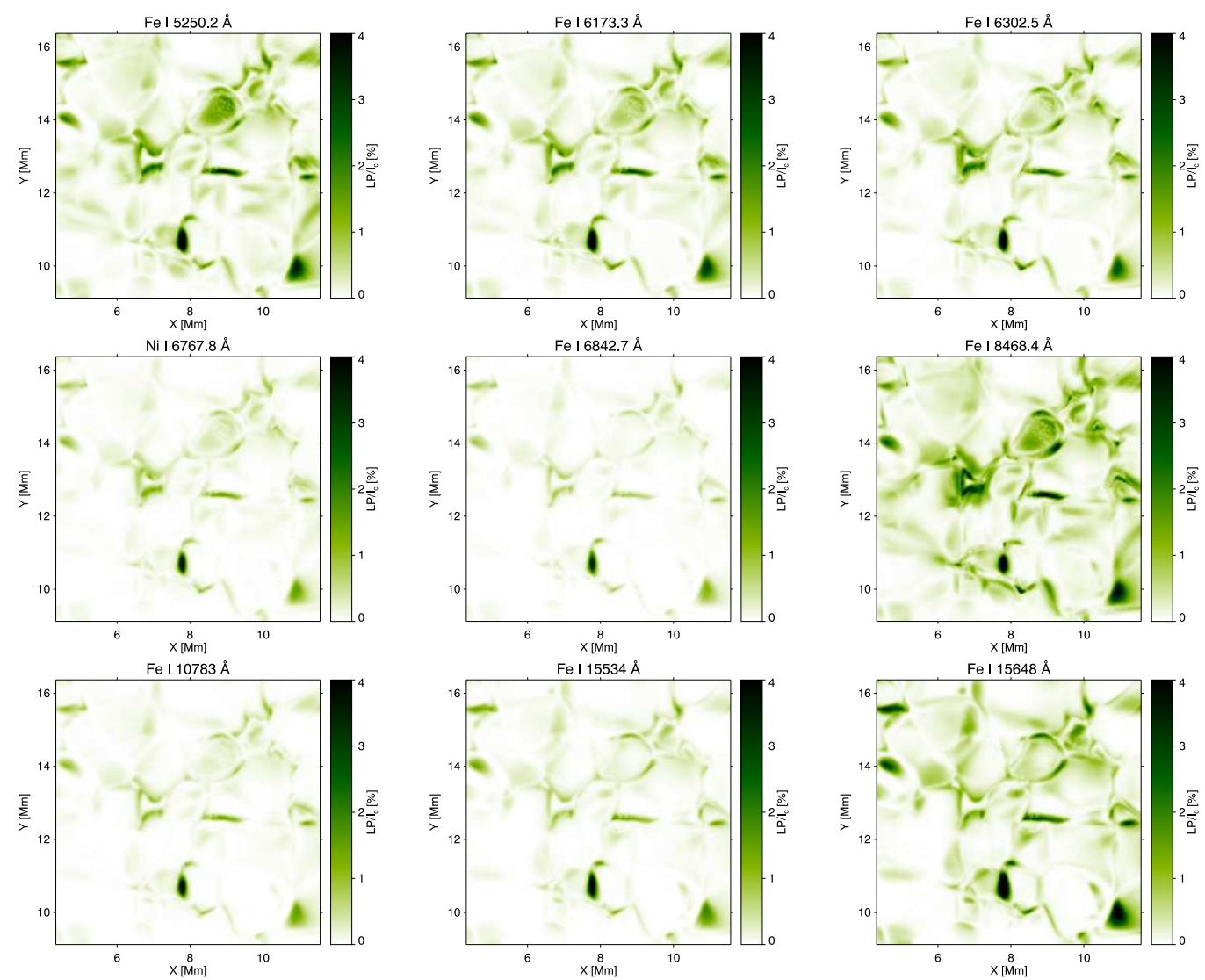

Fig. 8. Spatial distribution of maximum linear polarisation signals for the lines of interest. White areas indicate a low degree of polarisation, and darker regions designate the highest signals. The selected FOV corresponds to the highlighted area in Fig. 1 that is described in Fig. 6.
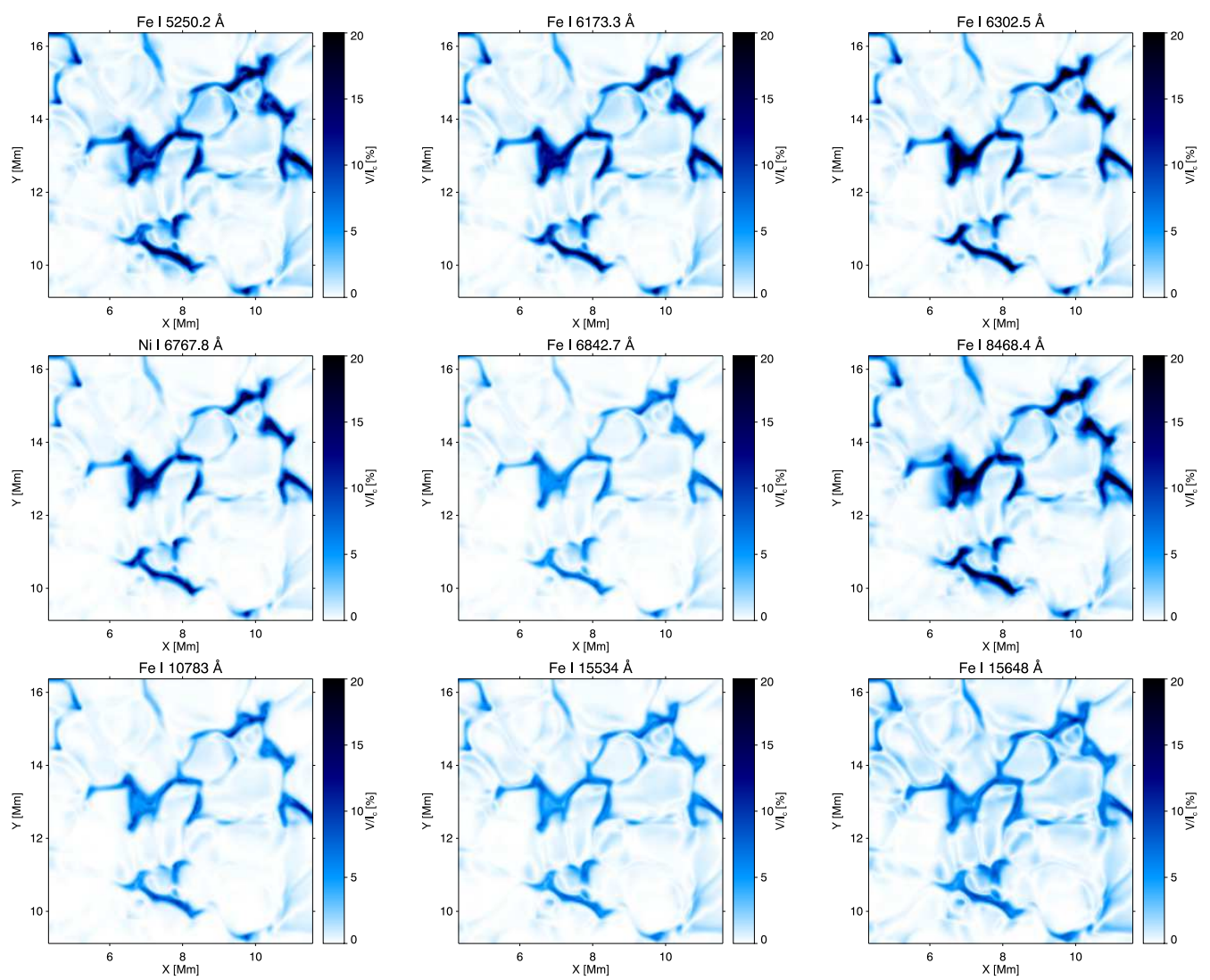

Fig. 9. Spatial distribution of maximum circular polarisation signals for the lines of interest. White areas indicate a low polarisation degree, and darker regions designate the largest amplitude. The selected FOV corresponds to the highlighted area in Fig. 1 that is described in Fig. 6. 

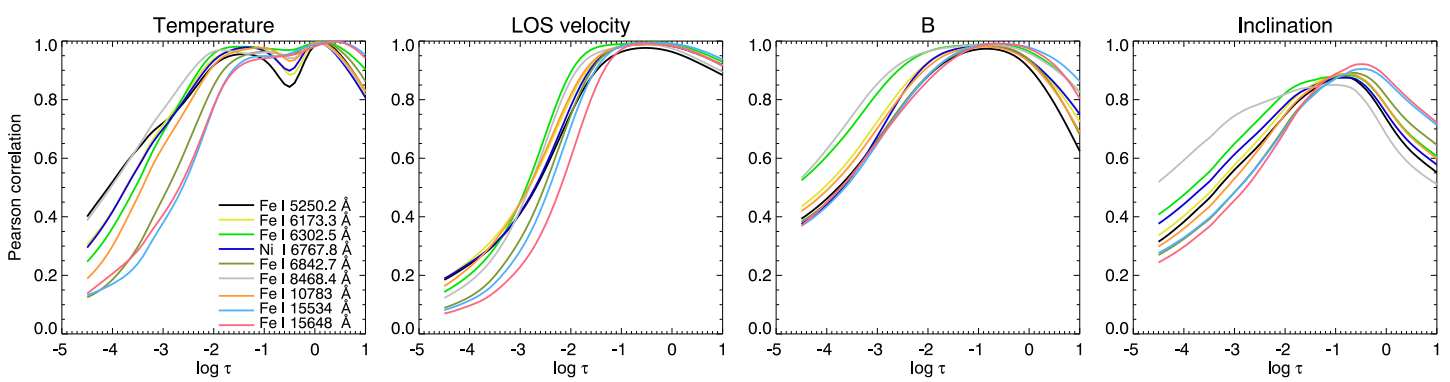

Fig. 10. Spatial correlation between the input atmosphere and the atmospheric parameters obtained from the inversion. From left to right: temperature, LOS velocity, field strength, and inclination. We computed the correlation between the input and the inferred atmospheric parameters at different optical depths.
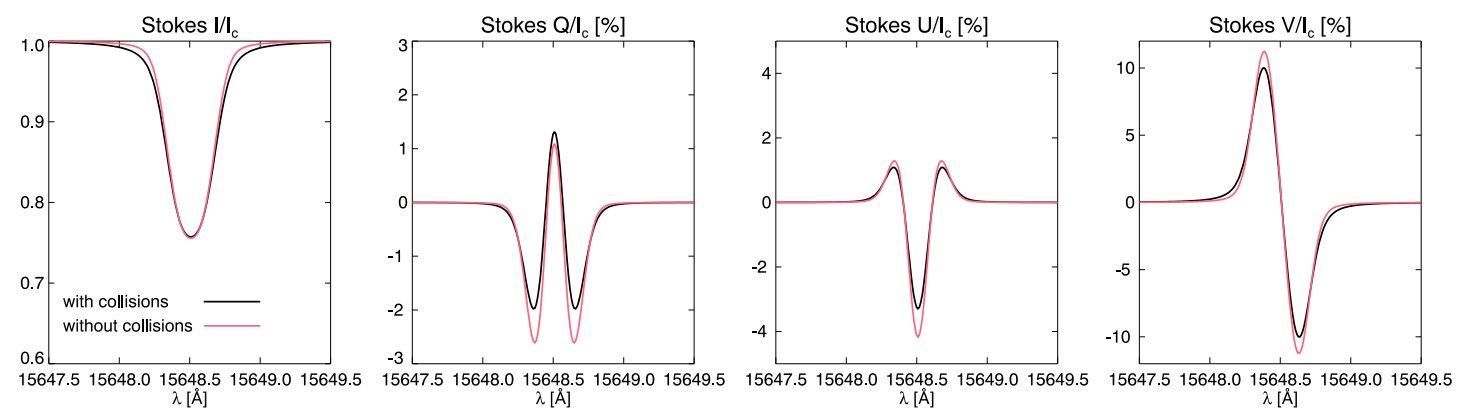

Fig. 11. Comparison between the synthetic profiles for the Fe I 15648 A transition when using the theory of Anstee \& O'Mara (1995) for collisions with neutral hydrogen (black) and the case where no broadening process is included (red). We use the HSRA atmosphere with a constant magnetic field strength of $300 \mathrm{G}, 45^{\circ}$ of inclination, and $70^{\circ}$ of azimuth.

for most of the transitions. The correlation at the deepest layers is also higher for the spectral lines above 1 micron, while the upper layers show the opposite behaviour with the visible and red transitions, obtaining a high correlation up to $\log \tau \sim-3$. Continuing with the LOS velocity (second panel from the left), all the candidates achieve high correlation values in the range of optical depths $\log \tau=[0,-1.5]$, indicating that the velocity stratification is covered at these heights with any of the spectral lines. Again, the infrared transitions above one micron show a high correlation at deeper layers that drops to low values at slightly lower layers than those of the visible lines. The situation is similar for the magnetic field strength, where the correlation in the range of optical depths $\log \tau=[0,-2.0]$ is high. However, in this case, it appears to be more evident that the iron lines in the visible and red part of the spectrum, $6302 \AA$ and $8468 \AA$, show a high correlation at moderately high layers. The situation for the magnetic field inclination is similar, where the correlation drops faster for the infrared (above 1 micron) spectral lines. However, it is also true that these spectral lines reach the highest correlation (at any height), which again indicates a high sensitivity to the transversal component of the magnetic field.

\section{Discussion}

\subsection{Effect of collisions with neutral hydrogen on the inversions of spectropolarimetric data}

We assumed so far that the theory presented in Anstee \& O'Mara (1995) should be included in the future when spectropolarimetric observations of the lines compared here are inverted. For this reason, we focused only on computing or updating the crosssection values for these transitions. However, it is worth showing the change in Stokes profiles when we include or exclude collisions with neutral hydrogen. Most importantly, however, we also wish to show the effect on the inferred atmospheric parameters when collisions are included or excluded during the inversion process.

We start with the effect on the polarimetric signals when the theory of Anstee \& O'Mara (1995) is assumed. We used the original HSRA atmosphere, adding a constant magnetic field strength of $300 \mathrm{G}, 45^{\circ}$ of inclination, and $70^{\circ}$ of azimuth. We show in Fig. 11 the results of the synthesis of the Fe I $15648 \AA$ transition, chosen as a reference spectral line for this discussion, when collisions with neutral hydrogen are included (black) or excluded (red). The intensity profile computed without collisions is narrower and slightly deeper, which produces polarimetric profiles with a larger amplitude. These differences will affect the inferred atmospheric parameters, and we wish to quantify which parameters are more affected.

We performed two inversions of the Stokes profiles synthesised with collisions (black in Fig. 11). In one case, we provided the code with the cross-section parameters, and the second case was without collisions. The results of the fit are shown in the top row of Fig. 12 with the original Stokes parameters in black, the results of the fit including collisions in blue, and in red that without considering collisions. The two fits are almost perfect; the one without collisions is less accurate. In the bottom row, we show the inferred atmospheric parameters using the same colour code. When collisions are included (blue), the fits match the original atmosphere very well. However, when the theory of Anstee \& O'Mara (1995) is not included in the fitting, the code has to reproduce the larger broadening of the intensity profile modifying the thermal properties of the atmosphere (see red). It raises the temperature above $\log \tau \sim-0.7$ and also adds a microturbulence enhancement of $1 \mathrm{~km} \mathrm{~s}^{-1}$. For the remaining physical parameters (we only show the LOS velocity and the magnetic field strength), the atmospheric values are identical for both inversions. These results indicate that if we do not consider 

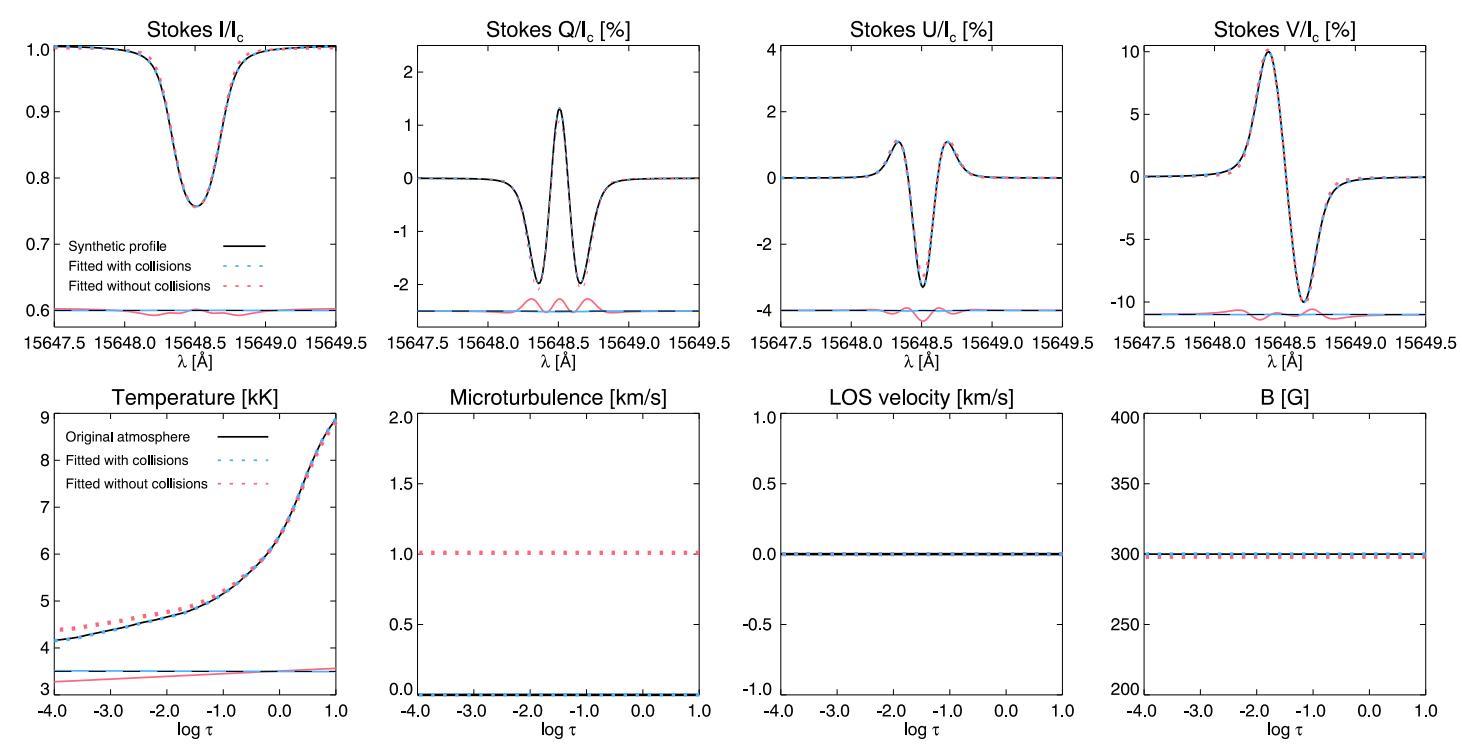

Fig. 12. Results for the inversion of the Fe I $15648 \AA$ synthetic profile presented in Fig. 11 in black (top row). This profile was synthesised considering collisions with $\mathrm{H}$. The effects of fitting this profile taking into account collisions with neutral hydrogen are plotted in blue, and those without considering collisions are shown in red. Bottom row: atmosphere that produced the input profiles (black) and the atmosphere inferred considering collisions with $\mathrm{H}$ (blue) and considering no collisions (red). We show the differences between the two studies at the bottom of the four Stokes parameters and the temperature panels.

the broadening due to collisions with neutral hydrogen when fitting observations, we modify the temperature and add an artificial microturbulence that will make our results less accurate.

\subsection{Suitability of the LTE approximation for photospheric lines}

We concentrated our efforts on comparing multiple spectral lines at identical conditions. These conditions mean solving the radiative transfer equation for the four Stokes parameters under the LTE approximation, which is widely used to analyse spectropolarimetric observations. However, several studies have found deviations from LTE for the photospheric lines presented here. The first work we are aware of is from Athay \& Lites (1972), but there are additional ones from (among others, Rutten \& Kostik 1982; Rutten 1988; Shchukina \& Trujillo Bueno 2001) or more recent publications including $3 \mathrm{D}$ radiative transfer computations (Holzreuter \& Solanki 2012, 2013, 2015; Smitha et al. 2020). We used the LTE approximation in this work because we aimed to compare a vast number of transitions, and LTE is the best tradeoff between accuracy and computational time. However, we plan to solve the radiative transfer equation for the transitions of interest in NLTE in future publications.

\section{Summary}

This work is the first step in a series of publications in which we plan to revise the literature searching for spectral line candidates, and we compare them using modern tools such as 3D realistic simulations. We dedicated some effort to extracting the atomic information of these lines and to computing the crosssection parameters due to collisions with $\mathrm{H}$. The second step is crucial to avoid mixing the line broadening induced by the temperature or plasma flows with that intrinsic of the atomic transition. Then, we compared at identical conditions the nine best candidate photospheric lines. We examined their properties using the HSRA atmosphere first, computing their maximum polarisation signals and their response to perturbations in the atmospheric parameters. The outcome of these studies indicated that four spectral lines stand out, Fe I 5250, 6302, 8468, and $15648 \AA$. They produce higher polarisation signals and with a broader height coverage. We then employed 3D numerical simulations to examine the spatial distribution of line core intensity and maximum polarisation signals. The results are similar to the previous study, with the four lines showing the largest amplitude. The Fe I $15648 \AA$ A line forms lowest in the atmosphere, while Fe I 5250,6302 , and $8468 \AA$ form at similar heights and above the previous line. We also included the results from the inversions of the synthetic data. The results confirmed what we found before, showing a good correlation with the input atmosphere in deeper layers for Fe I $15648 \AA$ and a better correlation at higher layers for the other three transitions.

\section{Recipe for optimised measurements of photospheric magnetic fields?}

We started this work with the aim to offer a recipe for the best spectral line for photospheric spectropolarimetry. However, creating such a recipe could seem like a challenge in view of the infinite options offered by the DKIST and EST telescopes. Most importantly, we admit that there is no definitive answer regarding a spectral line that can do everything, and the perfect recipe probably always requires more than just one ingredient. We therefore have four lines that outshine the rest in quiet-Sun conditions: Fe I 5250, 6302, 8468, and $15648 \AA$. They produce the strongest signals, show the largest RFs, and cover the broadest range of heights. The $15648 \AA$ line forms lower in the atmosphere. The opposite scenario holds for the other three; they extend higher in the photosphere but are less sensitive to deeper layers. The best recipe therefore appears to imply combining at least one of the first three spectral lines with the Fe I 15648 A to maximise our sensitivity to the atmospheric parameters in the broadest possible height range. It is exciting that the Fe I 5250, 6302 and $8468 \AA$ lines are more than 3000 (2000) $\AA$ apart, which means that we can choose one instead of the other based on what we wish to 
C. Quintero Noda et al.: Diagnostic capabilities of spectropolarimetric observations for understanding solar phenomena. I.

observe in a given spectral range. At the same time, we do not wish to leave the Fe I $6173 \AA$ transition out of the formula. It is a slightly worse than the other three visible-red transitions, but also has particular advantages. It is an isolated line with a clean continuum that makes it useful, for example, for space observations. Moreover, we should not exclude the Ni I $6767.8 \AA$ spectral line from the recipe. It was almost the most sensitive spectral line when we computed the RF to the LOS velocity, and although it produced low linear polarisation signals in the Bifrost simulation, the Stokes $V$ signals were comparable to those generated by the best spectral lines.

We strongly recommend combining any or several of these spectral lines with the Fe I $15648 \AA$ because it reaches lower than any of those transitions. It will also excel in quiet-Sun observations where the magnetic field is weakest.

Acknowledgements. C. Quintero Noda was supported by the EST Project Office, funded by the Canary Islands Government (file SD 17/01) under a direct grant awarded to the IAC on ground of public interest, and this activity has also received funding from the European Union's Horizon 2020 research and innovation programme under grant agreement No 739500. This work was supported by the Research Council of Norway through its Centres of Excellence scheme, project number 262622, and through grants of computing time from the Programme for Supercomputing. P. S. Barklem received financial support from the Swedish Research Council. The Centre for Earth and Space Research of University of Coimbra is funded by National Funds through FCT - Foundation for Science and Technology (project: UID/MULTI/00611/2019) and FEDER - European Regional Development Fund through COMPETE 2020 - Operational Programme Competitiveness and Internationalization (project: POCI-010145-FEDER-006922). This work has been funded by the Spanish Ministry of Science and Innovation through project ESP-2016-77548-C5-1-R, including a percentage from FEDER funds, and through the Centro de Excelencia Severo Ochoa grant SEV-2017-0709 awarded to the Instituto de Astrofísica de Andalucía in the period 2018-2022. D. Orozco Suárez also acknowledges financial support through the Ramón y Cajal fellowships. This work was supported by the ISAS/JAXA Small Mission-of-Opportunity program for novel solar observations and the JSPS KAKENHI Grant Number JP18H05234.

\section{References}

Anstee, S. D., \& O’Mara, B. J. 1995, MNRAS, 276, 859

Asplund, M., Grevesse, N., Sauval, A. J., \& Scott, P. 2009, ARA\&A, 47, 481

Athay, R. G., \& Lites, B. W. 1972, ApJ, 176, 809

Balthasar, H., \& Gömöry, P. 2008, A\&A, 488, 1085

Barklem, P. S., \& O’Mara, B. J. 1997, MNRAS, 290, 102

Barklem, P. S., Anstee, S. D., \& O'Mara, B. J. 1998, PASA, 15, 336

Barklem, P. S., Anstee, S. D., \& O’Mara, B. J. 2015, Astrophysics Source Code Library [record ascl:1507.007]

Barthol, P., Gandorfer, A., Solanki, S. K., et al. 2011, Sol. Phys., 268, 1

Beck, C., Fabbian, D., Rezaei, R., \& Puschmann, K. G. 2017, ApJ, 842, 37

Bello González, N., Danilovic, S., \& Kneer, F. 2013, A\&A, 557, A102

Bellot Rubio, L., \& Orozco Suárez, D. 2019, Liv. Rev. Sol. Phys., 16, 1

Bjørgen, J. P., Sukhorukov, A. V., Leenaarts, J., et al. 2018, A\&A, 611, A62

Cabrera Solana, D., Bellot Rubio, L. R., \& del Toro Iniesta, J. C. 2005, A\&A, 439, 687

Carlin, E. S., \& Bianda, M. 2017, ApJ, 843, 64

Carlsson, M., Hansteen, V. H., Gudiksen, B. V., Leenaarts, J., \& De Pontieu, B 2016, A\&A, 585, A4

Cheung, M. C. M., Rempel, M., Chintzoglou, G., et al. 2019, Nat. Astron., 3, 160

Collados, M., Martinez Pillet, V., Ruiz Cobo, B., del Toro Iniesta, J. C., \& Vazquez, M. 1994, A\&A, 291, 622

Collados, M., Lagg, A., Díaz Garcí, J. J., et al. 2007, in The Physics of Chromospheric Plasmas, eds. P. Heinzel, I. Dorotovič, \& R. J. Rutten, ASP Conf. Ser., 368, 611

Collados, M., López, R., Páez, E., et al. 2012, Astron. Nachr., 333, 872

Collados, M., Bettonvil, F., Cavaller, L., et al. 2013, Mem. Soc. Astron. It., 84, 379

da Silva Santos, J. M., de la Cruz Rodríguez, J., \& Leenaarts, J. 2018, A\&A, 620, A124

de la Cruz Rodríguez, J., Socas-Navarro, H., Carlsson, M., \& Leenaarts, J. 2012, A\&A, 543, A34

de la Cruz Rodríguez, J., Leenaarts, J., \& Asensio Ramos, A. 2016, ApJ, 830, L30 de la Cruz Rodríguez, J., Leenaarts, J., Danilovic, S., \& Uitenbroek, H. 2019, A\&A, 623, A74

Delbouille, L., Roland, G., \& Neven, L. 1973, Atlas photometrique du spectre solaire de [lambda] 3000 a [lambda] 10000 (Liege: Universite de Liege)

De Pontieu, B., Title, A. M., Lemen, J. R., et al. 2014, Sol. Phys., 289, 2733

Domingo, V., Fleck, B., \& Poland, A. I. 1995, Sol. Phys., 162, 1

Elmore, D. F., Lites, B. W., Tomczyk, S., et al. 1992, in Society of Photo-Optical Instrumentation Engineers (SPIE) Conference Series, eds. D. H. Goldstein, \& R. A. Chipman, Proc. SPIE, 1746, 22

Fossum, A., \& Carlsson, M. 2005, ApJ, 625, 556

Gafeira, R., Orozco Suárez, D., Milic, I., et al. 2021, A\&A, 651, A31

Gingerich, O., Noyes, R. W., Kalkofen, W., \& Cuny, Y. 1971, Sol. Phys., 18, 347 Gudiksen, B. V., Carlsson, M., Hansteen, V. H., et al. 2011, A\&A, 531, A154

Hansteen, V. H., Archontis, V., Pereira, T. M. D., et al. 2017, ApJ, 839, 22

Hansteen, V., Ortiz, A., Archontis, V., et al. 2019, A\&A, 626, A33

Harvey, J. W., Hill, F., Hubbard, R. P., et al. 1996, Science, 272, 1284

Holzreuter, R., \& Solanki, S. K. 2012, A\&A, 547, A46

Holzreuter, R., \& Solanki, S. K. 2013, A\&A, 558, A20

Holzreuter, R., \& Solanki, S. K. 2015, A\&A, 582, A101

Katsukawa, Y., del Toro Iniesta, J. C., Solanki, S. K., et al. 2020, SPIE Conf. Ser., 11447, 114470Y

Keil, S. L., Rimmele, T. R., Wagner, J., Elmore, D., \& ATST Team 2011, in Solar Polarization 6, eds. J. R. Kuhn, D. M. Harrington, H. Lin, et al., ASP Conf. Ser., 437, 319

Khomenko, E. V., Martínez González, M. J., Collados, M., et al. 2005, A\&A, 436, L27

Kosugi, T., Matsuzaki, K., Sakao, T., et al. 2007, Sol. Phys., 243, 3

Kramida, A., Ralchenko, Y., Reader, J., \& NIST ASD Team 2018, NIST Atomic Spectra Database (version 5.6.1), http://physics.nist.gov/asd

Kurucz, R. L., \& Bell, B. 1995, Atomic Line List (Cambridge, MA: Smithsonian Astrophysical Observatory)

Landi Degl'Innocenti, E., \& Landi Degl'Innocenti, M. 1977, A\&A, 56, 111

Landi Degl'Innocenti, E., \& Landolfi, M. 2004, in Polarization in Spectral Lines (Dordrecht: Kluwer Academic Publishers), Astrophys. Space Sci. Lib., 307

Leenaarts, J., Carlsson, M., Hansteen, V., \& Rouppe van der Voort, L. 2009, ApJ, 694, L128

Leenaarts, J., Pereira, T. M. D., Carlsson, M., Uitenbroek, H., \& De Pontieu, B. 2013, ApJ, 772, 89

Lites, B. W., Akin, D. L., Card, G., et al. 2013, Sol. Phys., 283, 579

Martínez González, M. J., Collados, M., Ruiz Cobo, B., \& Beck, C. 2008, A\&A, 477, 953

Martínez Pillet, V., Del Toro Iniesta, J. C., Álvarez-Herrero, A., et al. 2011, Sol. Phys., 268, 57

Mathew, S. K., Lagg, A., Solanki, S. K., et al. 2003, A\&A, 410, 695

Milić, I., \& van Noort, M. 2018, A\&A, 617, A24

Muglach, K., \& Solanki, S. K. 1992, A\&A, 263, 301

Müller, D., Marsden, R. G., \& St. Cyr, O. C., \& Gilbert, H. R., 2013, Sol. Phys., 285,25

Norton, A. A., Graham, J. P., Ulrich, R. K., et al. 2006, Sol. Phys., 239, 69

Pastor Yabar, A., Borrero, J. M., \& Ruiz Cobo, B. 2019, A\&A, 629, A24

Pesnell, W. D., Thompson, B. J., \& Chamberlin, P. C. 2012, Sol. Phys., 275, 3

Quintero Noda, C., Shimizu, T., de la Cruz Rodríguez, J., et al. 2016, MNRAS, 459, 3363

Quintero Noda, C., Shimizu, T., Katsukawa, Y., et al. 2017a, MNRAS, 464, 4534

Quintero Noda, C., Uitenbroek, H., Katsukawa, Y., et al. 2017b, MNRAS, 470, 1453

Quintero Noda, C., Uitenbroek, H., Carlsson, M., et al. 2018, MNRAS, 481, 5675

Ruiz Cobo, B., \& del Toro Iniesta, J. C. 1992, ApJ, 398, 375

Rutten, R. J. 1988, in The NLTE Formation of Iron Lines in the Solar Photosphere, eds. R. Viotti, A. Vittone, \& M. Friedjung, Astrophys. Space Sci. Lib., 138, 185

Rutten, R. J., \& Kostik, R. I. 1982, A\&A, 115, 104

Scherrer, P. H., Bogart, R. S., Bush, R. I., et al. 1995, Sol. Phys., 162, 129

Schmidt, W., von der Lühe, O., Volkmer, R., et al. 2012, Astron. Nachr., 333, 796

Schou, J., Scherrer, P. H., Bush, R. I., et al. 2012, Sol. Phys., 275, 229

Shchukina, N., \& Trujillo Bueno, J. 2001, ApJ, 550, 970

Smitha, H. N., \& Solanki, S. K. 2017, A\&A, 608, A111

Smitha, H. N., Holzreuter, R., van Noort, M., \& Solanki, S. K. 2020, A\&A, 633, A 157

Solanki, S. K. 2003, A\&ARv, 11, 153

Solanki, S. K., Barthol, P., Danilovic, S., et al. 2010, ApJ, 723, L127

Solanki, S. K., del Toro Iniesta, J. C., Woch, J., et al. 2020, A\&A, 642, A11

Stenflo, J. O. 1973, Sol. Phys., 32, 41

Sukhorukov, A. V., \& Leenaarts, J. 2017, A\&A, 597, A46

Tsuneta, S., Ichimoto, K., Katsukawa, Y., et al. 2008, Sol. Phys., 249, 167

Štěpán, J., \& Trujillo Bueno, J. 2016, ApJ, 826, L10 


\section{Appendix A: Fe। $15652.874 \AA$}

The pair of Fe I infrared lines at 15648 and $15652 \AA$ has been extensively used in the literature for polarimetric measurements since the early 1990s. Among many other works, we have the series of works entitled Infrared lines as probes of solar magnetic features that started with Muglach \& Solanki (1992), or the publications of, for instance, Mathew et al. (2003), Khomenko et al. (2005), Bello González et al. (2013), and Beck et al. (2017). If we examined the atomic information for both lines, presented in Table 2, the second line cannot be treated assuming LScoupling. We therefore excluded it from Table 1 in Sect. 2. However, we plan to describe how it can be appropriately included in the SIR code for future inversions. The same modifications can also probably be implemented in any of the new codes, such as SNAPI (Milić \& van Noort 2018), StiC (de la Cruz Rodríguez et al. 2019), or FIRTEZ-dz (Pastor Yabar et al. 2019).

The effective Landé factor of the Fe I $15652 \AA$ transition should be evaluated considering a $J-K$ coupling (also known as $J_{1}-l$ coupling, see Landi Degl'Innocenti \& Landolfi 2004). Paraphrasing the authors, we have that another case where it is possible to find an analytical expression for the Landé factor is the case of the so-called $J_{1}-l$ coupling. In this coupling scheme, a parent level of orbital angular momentum $L_{1}$ and spin $S_{1}$ couples its total angular momentum $J_{1}$ with the orbital angular momentum $l$ of a further electron, to give an angular momentum $K$ which in its turn couples with the electron spin to give the total angular momentum $J$. To evaluate the Landé factor for this type of transition, we therefore need to provide the quantum numbers $L_{1}, S_{1}$, and $J_{1}$ of the parent level, the quantum numbers of the last electron $l$ and $s=1 / 2$, the orbital angular momentum of the coupling $K$, and the total angular momentum $J$.
In the case of SIR, we need the spectroscopic information $7 D$ $5.0-(6 D 4.5) f 2 k 4$. The first is the LS-coupling description of the lower level and the second is the $J_{1}-l$ coupling information of the upper level. Every time the code finds a parenthesis in the atomic information, as for the upper level, it reads the information inside as the parent term, and it considers that the $J-K$ coupling is defined. In this example, the configuration of the upper level says $(6 D 4.5) f 2 k 4$, which means that $S_{1}=2.5, L_{1}=2$ (because the angular momentum is $D$ ), $J_{1}=4.5, l=3$ (because the angular momentum of the last electron is $f$ ), $s=1 / 2$ (we do not need to provide it because the spin of the additional electron is always taken as $1 / 2$ ). Then, the orbital angular momentum of the coupling $K$ is given by the NIST database as $K=7 / 2$. However, in SIR, following the previous convention, we use characters, similar to the linear angular momentum, with $p=0.5$, $f=1.5, h=2.5, k=3.5, m=4.5, o=5.5, r=6.5, t=7.5$, $u=8.5, v=8.5$, and $w=9.5$. This means that in the input file for the code, we write $(6 D 4.5) f 2 k$. Lastly, the total angular momentum is $J=4$.

Gathering all this information, we can use the following equation (see Landi Degl'Innocenti \& Landolfi 2004):

$g_{J_{1} l}=1+\gamma(J, 1 / 2, K)+\gamma(J, K, 1 / 2) \gamma\left(K, J_{1}, l\right) \gamma\left(J_{1}, S_{1}, L_{1}\right)$,

where $\gamma$ is defined as

$\gamma(A, B, C)=\frac{A(A+1)+B(B+1)-C(C+1)}{2 A(A+1)}$.

For the Fe I $15652 \AA$ infrared transition, we have then that $g_{J_{1} l}=$ 2.097. We modified the SIR code to accept any transition in LS or $J-K$ coupling. Anyone interested in using the new version or knowing more about how we did it is more than welcome to contact us. 